\title{
Bipolariton coupling in biexciton optical decay: Degenerate and nondegenerate polariton emissions in $\mathrm{CuCl}$
}

\author{
Eiji Tokunaga, ${ }^{1, *}$ Kazuyoshi Kurihara ${ }^{2, \dagger}$ Motoyoshi Baba, ${ }^{1}$ Yasuaki Masumoto, ${ }^{3, \ddagger}$ and Masahiro Matsuoka ${ }^{4, \S}$ \\ ${ }^{1}$ Institute for Solid State Physics, University of Tokyo, 5-1-5 Kashiwanoha, Kashiwa, Chiba 277-8581, Japan \\ ${ }^{2}$ Special Research Laboratory for Optical Science, Kanagawa Academy of Science and Technology, 3-2-1 Sakado, \\ Takatsu-ku, Kawasaki 213-0012, Japan \\ ${ }^{3}$ Single Quantum Dot Project, ERATO, JST, Tokodai 5-9-9, Tsukuba 300-2635, Japan \\ ${ }^{4}$ Department of Physics, Faculty of Science, Kumamoto University, 2-39-1 Kurokami, Kumamoto 860-0862, Japan
}

(Received 12 June 2000; revised manuscript received 17 January 2001; published 3 July 2001)

Radiative decay of biexcitons wave-vector $(\mathbf{K})$ selectively generated in a low- $K$ region $\left(0 \leqslant|\mathbf{K}| \leqslant 2 k_{0}\right)$ is studied in $\mathrm{CuCl}$, where $2 k_{0}$ is the biexciton wave number of degenerate two-photon excitation, and the bipolariton nature of low- $K$ biexcitons is experimentally demonstrated. All the radiative relaxation channels of low- $K$ biexcitons are clarified, which dissociate into two lower-branch polaritons and into upper- and lowerbranch polaritons yielding nearly degenerate and nondegenerate pair emission lines, respectively. The threedimensional scattering geometry allows for simultaneous observation of both degenerate and nondegenerate lines. Nearly degenerate two-polariton emissions have larger intensities than nondegenerate emissions. Completely degenerate emissions from $\mathbf{K}=2 \mathbf{k}_{0}$ biexcitons have the largest intensity, reflecting singularity of the joint density of polariton states. These features are in remarkable contrast with the resonant two-photon absorption process of biexcitons, which shows resonance enhancement not at the degenerate two-photon energy but at the exciton resonance. In spite of the larger transition probability, nearly degenerate emissions have a negligible contribution to the emission band arising from thermally distributed biexcitons. The dispersion curve of the $Z_{1,2}\left(Z_{3}\right)$-exciton lower(upper)-branch polariton is determined from the $K$ dependence of the emission energies. The observed emission lines are all unambiguously assigned and the relative intensities of the simultaneously observed lines are compared with a biexciton radiative decay theory based on the bipolariton model. The energy dependence of the transition probability of the observed biexciton radiative decay channels is quantitatively reproduced by the theory, verifying that the biexciton radiative decay rate is determined by the exciton components in final-state polaritons (Hopfield coefficients) through the exciton-exciton Coulomb interaction. The radiative lifetimes of $K \simeq 0$ biexcitons are found to be shorter than those of $K$ $\simeq 2 k_{0}$ biexcitons as expected from the theory. The lifetimes of low- $K$ biexcitons are strongly affected by the coupling with final two-polariton states, leading to a $20 \%$ reduction of the lifetimes compared with the case of no polariton effects.

DOI: 10.1103/PhysRevB.64.045209

PACS number(s): 71.35.-y, 71.36.+c, 78.55.Hx, 42.65.Yj

\section{INTRODUCTION}

The optical processes of biexcitons (excitonic molecules) ${ }^{1}$ in bulk direct-gap semiconductors are mediated by intermediate polariton states. ${ }^{2}$ This offers challenging problems because both exciton-exciton ${ }^{3-5}$ and exciton-photon interactions should be nonperturbatively treated. In terms of the polariton, the problems are restated as follows. Polariton eigenstates are coupled harmonic oscillators of electromagnetic and mechanical waves due to resonant light-matter interaction, but they break down if nonlinearity arises through higher-order interactions. ${ }^{6}$ In cavity polaritons ${ }^{7}$ too, this is one of the intriguing issues. ${ }^{8}$ Recently polariton-polariton interaction through exciton-exciton interaction is actively studied both experimentally ${ }^{9}$ and theoretically. ${ }^{10-12}$ This interaction leads to the formation of biexciton bound states ${ }^{1}$ in the strong interaction limit, not only in bulk semiconductors but also in cavity polaritons as identified recently. ${ }^{13,14}$

The conventional theoretical approach to describe the biexciton optical processes is the giant oscillator strength (GOS) model, ${ }^{15,16}$ which treats the interaction as an optical transition between the biexciton and exciton states. The model did not include polariton effects originally and thus has undergone several modifications to account for polariton effects. ${ }^{17,18}$ Another approach is a bipolariton (BP) model, ${ }^{19}$ which treats the exciton-exciton interaction rigorously in the biexciton formation and dissociation processes. The BP model involves polariton effects naturally and is considered to be the most precise model that is presently available.

Polariton effects of biexciton optical processes have not been clearly demonstrated so far. Here we use polariton effects not for the modification of the signal energy, which is determined by the polariton dispersion and already verified in numerous experiments, ${ }^{20-23}$ but for the modification of the signal intensity due to intermediate polariton states, which is relevant to Hopfield coefficients, i.e., exciton and photon components in polaritons. ${ }^{2}$ In order to study polariton effects in biexciton optics, there are two important aspects to note. First, the effects manifest themselves in spontaneous processes and second, biexcitons near $K_{m}=0$, where $K_{m}$ is a biexciton wave number, are most suitable to observe the effects.

The first important aspect is stated as follows. A variety of attractive optical phenomena associated with biexcitons have been reported such as two-photon absorption, ${ }^{1,15}$ stimulated emissions, ${ }^{24}$ four-wave mixing ${ }^{25-27}$ optical 
bistability, $^{28}$ optical stark shift, ${ }^{29}$ optical soliton propagation, ${ }^{30}$ etc. In these stimulated polariton scattering regimes, the efficiency of the processes is rather insensitive to the polariton eigenstates involved, ${ }^{31,32}$ because Hopfield coefficients combine with the corresponding polariton density of states. The process that explicitly depends on the Hopfield coefficients will be observed in the spontaneousscattering regime. ${ }^{32}$

Second, according to the BP model, biexciton states are more precisely described by bipolaritons if the wave numbers $k$ of the constituent excitons are close to the polariton resonance $k \simeq \sqrt{\varepsilon_{b}} \omega_{T} / c$, where $\omega_{T}$ is an exciton resonant frequency and $\varepsilon_{b}$ is a background dielectric constant. In this respect, biexcitons near $K_{m}=0$ are most suitable to study polariton effects (bipolariton coupling) because $K_{m} \simeq 0$ biexcitons decay into polaritons that are close to the polariton resonance. Thus polariton modification of biexcitons should be large as expected from the BP model. Further, while high$K_{m}$ biexcitons suffer from intraband relaxation processes before radiative decay, ${ }^{33,34} K_{m} \simeq 0$ biexcitons are free from them because there would be no final state after phonon emission in the biexciton band ${ }^{35}$ and the momentum relaxation rate by defect scattering is negligibly small owing to the near-zero momentum. Since the relaxation channels of $K_{m} \simeq 0$ biexcitons are limited to radiative decay, polariton effects can be observed without interference with other effects.

For the two reasons above, the spontaneous decay of $K_{m}$ $\sim 0$ biexcitons is the key to the understanding of bipolariton coupling, but their optical processes are not fully explored. In this field, $\mathrm{CuCl}$ has been studied in greatest detail as a prototypical material for exciton-polariton and biexciton physics ${ }^{1}$ because the ground-state exciton is the nondegenerate, isotropic $Z_{3}$ exciton, different from other zinc-blende semiconductors having the $Z_{1,2}$ exciton as the lowest state, ${ }^{36,37}$ and the binding energies of the exciton and biexciton are large $\left(\epsilon^{x} \simeq 200 \mathrm{meV}\right.$ and $\epsilon^{m} \simeq 32 \mathrm{meV}$, respectively). In regard to absorption, polarization spectroscopy was applied to obtain the two-photon absorption line width for $K_{m}=0$ biexcitons in $\mathrm{CuCl}^{31}$ where polariton effects are studied but not pronounced because of the stimulated process. For radiative decay, emissions from low- $K_{m}$ biexcitons in $\mathrm{CuCl}$ were observed using degenerate two-photon excitation and the $K_{m}$ dependence of emission energy was obtained by angle tuning of the two beams, ${ }^{38}$ but the energy range studied was not wide and emissions from the exact $K_{m}=0$ biexcitons were not reported.

In order to explain inverse-exciton series recently observed in $\mathrm{CuCl}$, a rigorous theory of biexciton optical decay has been developed on the BP model, ${ }^{39}$ where the radiative decay is nonperturbatively treated as the spontaneous, resonant dissociation of biexcitons into two polaritons. The relevant interaction is the exciton-exciton interaction and the transition probability is determined by the exciton components in final-state polaritons and by a precise structure of the biexciton wave function. The theory has been successfully applied to the analysis of inverse-polariton series in $\mathrm{CuCl},{ }^{40}$ but the theory has not been tested for the most domi- nant decay channels into polaritons associated with the ground-state exciton.

One of the difficulties for testing the theory is the overlap of the absorption and emission processes. As long as nearly degenerate two-photon excitation of biexcitons is employed, both incident and emitted polaritons are ground-state exciton polaritons, thus the emissions tend to be masked by the scattering of the incident light. This is avoided only if the threedimensional energy-momentum conservation ${ }^{1,41}$ is utilized. In fact, one can observe nearly degenerate polariton emission lines on both sides of the excitation laser without overlap, ${ }^{42}$ as was studied in detail by $\mathrm{Vu}$ et $a$ al. $^{43}$ and by Itoh and Suzuki. ${ }^{38}$ Since then, however, there has been no more detailed work on these emissions. Completely degenerate twopolariton emissions are the reverse process of degenerate two-photon absorption. To the best of our knowledge, there has been no clear observation of them, probably because nondegenerate excitation of biexcitons is needed as demonstrated in this paper. This process is distinguished from other emission processes because it is expected to reflect the van Hove singularity of the joint density of polariton states (JDPS). ${ }^{44}$

In this paper, we study two polariton emissions from low$K_{m}$ biexcitons in $\mathrm{CuCl}$, involving both upper- and lowerbranch polaritons and both one- and three-dimensional scattering conditions. Here, "low $K_{m}$ ", stands for $\left|\mathbf{K}_{m}\right|$ $\leqslant 2 k_{0}$ ("high $K_{m}$ ", for $\left.\left|\mathbf{K}_{m}\right| \geqslant 2 k_{0}\right)$, where $2 k_{0} \quad(=0.89$ $\left.\times 10^{6} \mathrm{~cm}^{-1}\right)$ is the wave number of biexcitons produced by degenerate two-photon excitation in $\mathrm{CuCl}$. (Nearly) degenerate two-polariton emissions are observed both from $\mathbf{K}_{m} \simeq 0$ and $\mathbf{K}_{m} \simeq 2 \mathbf{k}_{0}$ biexcitons. From this study we give a comprehensive view of the optical process of optically accessible low- $K_{m}$ biexcitons and test the radiative decay theory by rigorously comparing the emission intensities.

The description of experimental methods in Sec. II is followed by the experimental results in Sec. III. In Sec. III A, nondegenerate two-polariton emission spectra are presented, which are due to the biexciton optical decay into upper- and lower-branch polaritons in the one-dimensional scattering geometry. In Sec. III B, emission spectra of both collisionally and $\mathbf{K}_{m}$-selectively excited biexcitons are presented in the three-dimensional scattering geometry. Nearly degenerate two-polariton emission spectra of both $\mathbf{K}_{m} \simeq 0$ and $\mathbf{K}_{m}$ $\simeq 2 k_{0}$ biexcitons are shown. In Sec. IV, from the emission energies as a function of $K_{m}$, the polariton dispersion is determined in a wide energy region to clarify all the radiative decay channels of $K_{m} \sim 0$ biexcitons. The origins of all the emission lines are unambiguously identified. In Sec. V, the biexciton radiative decay theory on the BP model is experimentally verified with respect to the polariton branches associated with the ground-state exciton. High efficiency of degenerate emission processes due to bipolariton coupling is also demonstrated and the difference between absorption and emission processes is clarified. In Sec. VI, the $K_{m}$ dependences of the biexciton decaytime is precisely calculated, which is consistent with all the experimental results.

\section{EXPERIMENT}

We have measured biexciton emissions in $\mathrm{CuCl}$ in a low$K_{m}$ region by nondegenerate two-photon resonant excitation 


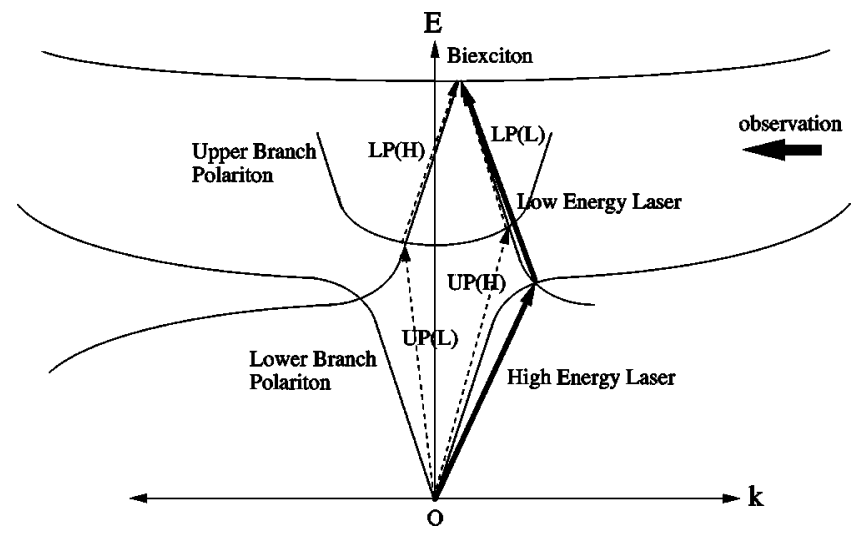

FIG. 1. The schematic of the excitation (thick solid arrow) of biexcitons near $K_{m}=0$ and the emissions (dashed arrow) shown with respect to the biexciton and polariton-dispersion curves. To show correctly the directions of the polariton wave vectors under the momentum conservation, the upward arrows are used for both excitation and emission processes. The $\operatorname{LP}(H)$ and $\operatorname{UP}(H)$ emissions are emitted in the observation direction while the $\operatorname{LP}(L)$ and $\mathrm{UP}(L)$ emissions are observed by internal reflection on the back surface.

of biexcitons. ${ }^{33}$ In Fig. 1, the schematic of the onedimensional biexciton resonant polariton-polariton scattering process in the antiparallel excitation case is shown with respect to the dispersion curves of biexcitons and polariton branches associated with the ground-state $Z_{3}-1 s$ exciton in $\mathrm{CuCl}$. The intersection of the curves indicates the polariton states that satisfy the energy- and momentum-conservation condition. Biexcitons are resonantly excited via two lowerbranch polaritons, and the resonant dissociation processes into upper- and lower-branch polaritons and into two lowerbranch polaritons are observed. The latter process can be observed in the three-dimensional scattering geometry as depicted for the case of collinear excitation of $2 k_{0}$ biexcitons in Fig. 2.

The light source is a high-repetition-rate synchronized two-color tunable picosecond uv laser as shown in Fig. 3. The high-repetition picosecond pulses are important both for high spectral resolution and for satisfying the weakexcitation condition to avoid higher-order nonlinearities due to reexcitation of biexcitons. ${ }^{42}$ The outputs of a cw modelocked YAG (yttrium aluminum garnet) laser and two synchronously pumped dye (Rhodamine 6G) lasers are frequency summed in $\mathrm{LiIO}_{3}$ crystals to deliver two-color picosecond uv pulses with $375-395 \mathrm{~nm}$ tunability, $8 \mathrm{ps}$ duration, $0.05 \mathrm{~nm}$ spectral width, $82-\mathrm{MHz}$ repetition, and 30 $\mathrm{mW}$ maximum average power for each beam. The two laser pulses are synchronized within 1 ps. The output power is increased by 15 times that in Ref. 33 by replacing a $9-\mathrm{W}$ YAG laser and a doubling crystal $\mathrm{KTiOPO}_{4}$ by a $15-\mathrm{W}$ YAG laser (Spectron) and a temperature-controlled $\mathrm{LiB}_{3} \mathrm{O}_{5}$ crystal, respectively. The improvement in the power of the light source by more than one order of magnitude is crucial for the observation of the low-intensity emissions from $K_{m} \sim 0$ biexcitons.

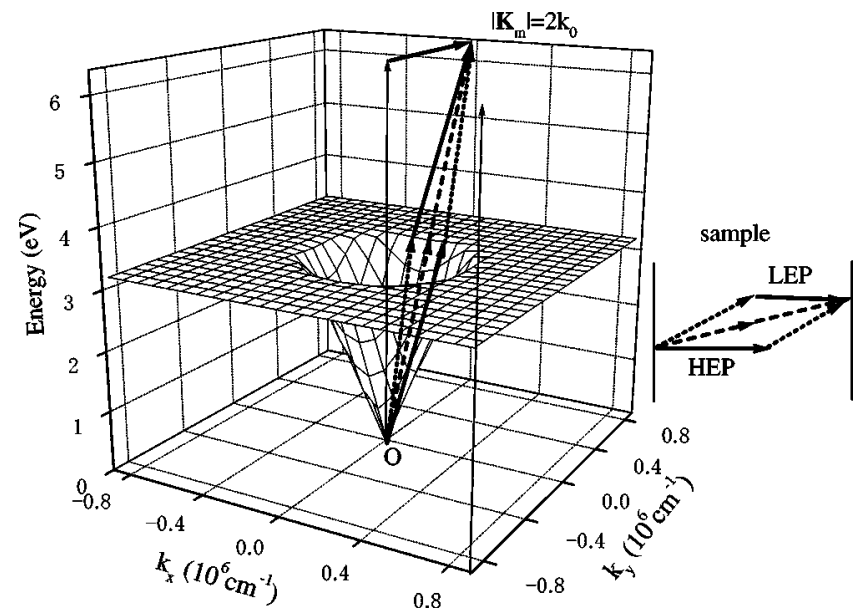

FIG. 2. The biexciton optical decay into the lower-polariton branch in $\mathrm{CuCl}$ under the three-dimensional energy- and momentum-conservation condition. When biexcitons are excited at $2 k_{0}$ by degenerate two-photon excitation (dashed arrows), the LEP and HEP lines (solid arrows) are observed at a finite angle from the excitation beam. The observable decay paths change continuously with the observation angle.

Platelets of $\mathrm{CuCl}$ single crystal (thickness of 10 $-20 \mu \mathrm{m})$ grown from gas phase are immersed in superfluid $\mathrm{He}$ at $2 \mathrm{~K}$ in a strain-free condition. Biexcitons of $\mathbf{K}_{m}$ $=\mathbf{k}_{1}^{(0)}+\mathbf{k}_{2}^{(0)}$ are excited by tuning one beam to energy lower than the degenerate two-photon resonant energy, $3.186 \mathrm{eV}$, and the other to higher energy keeping their sum constant $\left(E_{m}=6.372 \mathrm{eV}\right.$, biexciton energy). For antiparallel excitation, samples are illuminated by counterpropagating twocolor beams and emissions are collected on the low-energy laser side as shown in Fig. 4. For collinear excitation, samples are illuminated by collinear two-color beams with the wave vectors $\mathbf{k}_{1}^{(0)}$ and $\mathbf{k}_{2}^{(0)}\left(\mathbf{k}_{1}^{(0)} \| \mathbf{k}_{2}^{(0)}\right)$ at a finite incidence angle, and emissions normal to the sample surface are collected on the illumination side (backward scattering geometry). For both excitation configurations, two beams are superposed in a sample both spatially and temporally with $f=200 \mathrm{~mm}$ lenses and with an optical delay, respectively, and made left- and right-circularly polarized in a sample to satisfy the polarization selection rule ${ }^{1}$ and to avoid single-

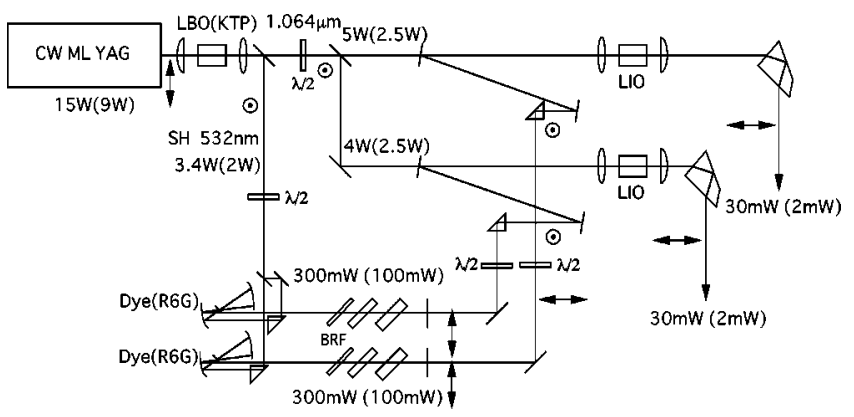

FIG. 3. High-repetition synchronized two-color tunable picosecond uv laser. The powers are improved from those in the previous paper (Ref. 33) shown in the parentheses. BRF, birefringent filter; $\lambda / 2$, half-wave plate; $\leftrightarrow$, horizontal polarization; $\odot$, vertical polarization. 


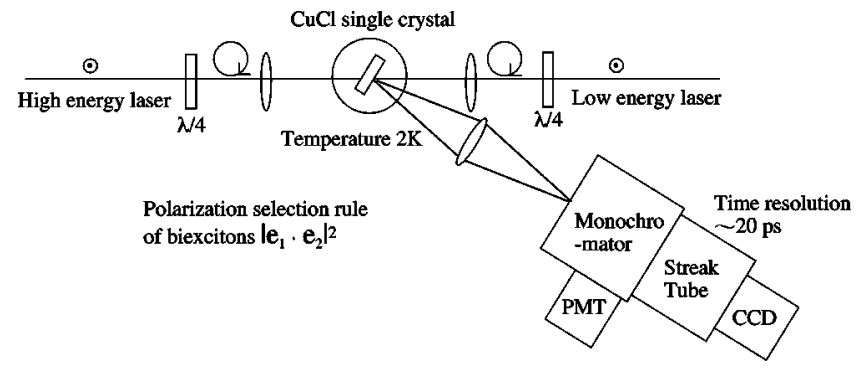

FIG. 4. Experimental setup with counterpropagating excitation beams and a monochromator system.

beam excitation of biexcitons. The excitation intensity is $\$ 500 \mathrm{~kW} / \mathrm{cm}^{2}$.

Time-integrated emission spectra are taken through a 50-cm monochromator (Chromex) with a photomultiplier tube at one port as shown in Fig. 4. At the other port of the monochromator, emissions are time resolved with a streak camera (Hamamatsu) and detected with an electrically cooled Si charge-coupled device (Princeton). The time resolution of the system is about $20 \mathrm{ps}$.

\section{RESULTS}

\section{A. One-dimensional scattering}

Figure 5 shows biexciton emission spectra for the wave numbers from $K_{m}=0$ to $1.6 k_{0}$ by nondegenerate antiparallel two-photon excitation in the one-dimensional scattering geometry. For $K_{m}=0$, weak emission lines denoted by UP (upper polariton) and LP (lower polariton) are observed at 3.228 $\mathrm{eV}$ and $3.145 \mathrm{eV}$, respectively. ${ }^{45}$ They are originating from upper- and lower- branch polaritons (UBP's and LBP's) split from $K_{m}=0$ biexcitons. This is the first observation, to the best of our knowledge, of UP and LP pair emissions from $K_{m}=0$ biexcitons in $\mathrm{CuCl}^{38,46}$ Their intensities are much weaker than that of the $M_{T}$ emissions observed by degenerate two-photon excitation of biexcitons ${ }^{42}$ nearly by two orders of magnitude. The extremely weak intensity is considered to be the reason for the absence of earlier observation. As $K_{m}$ is shifted from 0, the UP lines split into the $\mathrm{UP}(H)$ and $\operatorname{UP}(L)$ lines and the LP lines split into the $\operatorname{LP}(H)$ and $\mathrm{LP}(L)$ lines, because degeneracies of the polariton energies are lifted for $K_{m} \neq 0$ as displayed in Fig. 1. In the present configuration, the $\mathrm{UP}(H)$ and $\mathrm{LP}(H)$ lines are emitted in the observed direction, while the $\operatorname{UP}(L)$ and $\operatorname{LP}(L)$ lines are in the opposite direction and observed by internal reflection on the rear surface.

The $\operatorname{UP}(H)$ and $\operatorname{LP}(L)$ lines are pair-polariton emission lines. As their energy difference increases with $K_{m}$, their intensities decrease and are not detectable for $K_{m}>0.05$ $\times 10^{6} \mathrm{~cm}^{-1}$. The other pair lines of $\operatorname{UP}(L)$ and $\operatorname{LP}(H)$ behave oppositely with $K_{m}$. This intensity change reflects bipolariton coupling in the biexciton radiative decay as will be discussed in Sec. V. In addition, the UP and LP linewidths increase as their energy difference increases. Their full width at half maximum (FWHM) amounts to $3-4 \mathrm{meV}$ at the observed highest UP energy $\simeq 3.25 \mathrm{eV}$ (lowest LP energy $\simeq 3.13 \mathrm{eV}$ ). This mainly reflects the UBP scattering rates.

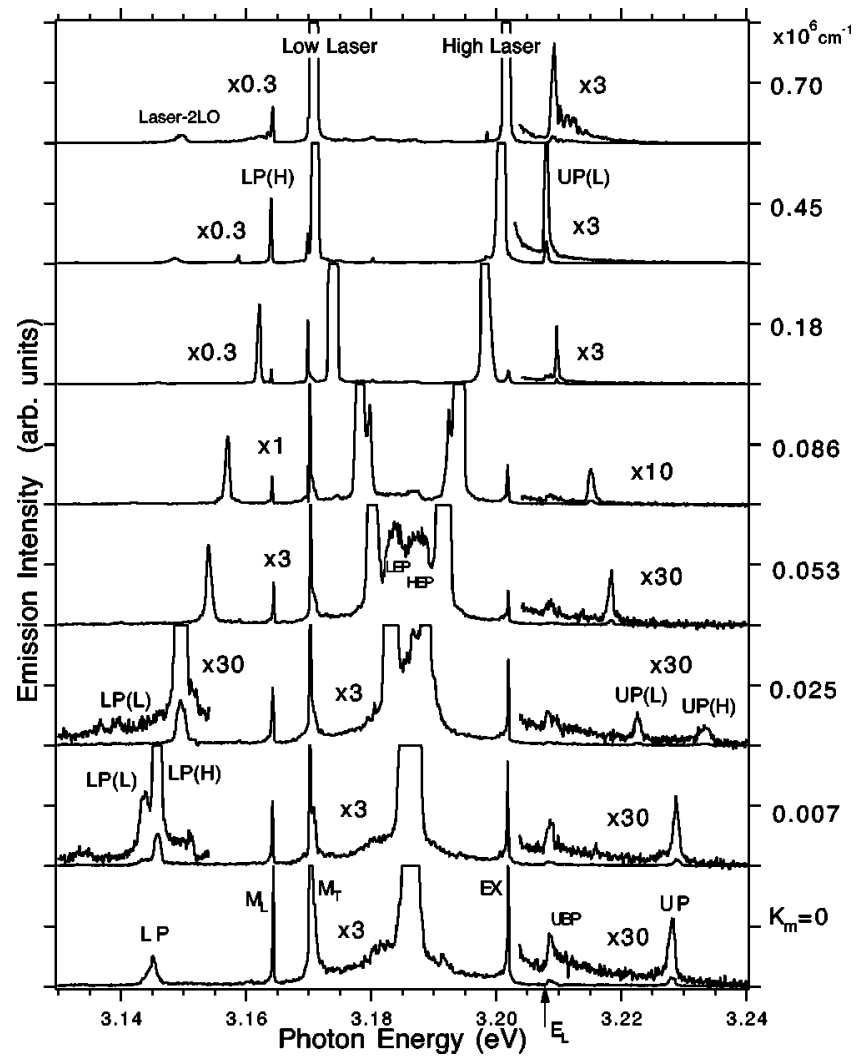

FIG. 5. Biexciton emission spectra for the wave numbers from $K_{m}=0$ to $0.70\left(\times 10^{6} \mathrm{~cm}^{-1}\right)$. The $\operatorname{LP}(L, H), \mathrm{UP}(L, H)$ emissions are from $k_{1}^{(0)}-k_{2}^{(0)}$ biexcitons and the $M_{T}, M_{L}$, EX, LEP, and HEP emissions are from $\simeq k_{1}^{(0)}+k_{2}^{(0)}$ biexcitons. The longitudinal exciton energy is denoted by $E_{L}$. The emissions labeled by UBP are attributed to polaritons distributed in the upper-polariton branch as a result of the intraband relaxation of the UP polaritons.

Their estimated values are larger than previously observed ${ }^{47}$ because the UBP energy is much higher than before. Detailed analysis about the width will be given separately. ${ }^{48}$

For $K_{m}=0.45 \times 10^{6} \mathrm{~cm}^{-1}\left(\simeq k_{0}\right)$, the energies of $\operatorname{UP}(L)$ and $\operatorname{LP}(H)$ are the same as the longitudinal exciton (marked by $E_{L}$ ) and $M_{L}$ energies, respectively, because biexcitons decay into the bottom of the UBP dispersion curves. Above $K_{m}=k_{0}$, the line shapes of $\operatorname{UP}(L)$ and $\operatorname{LP}(H)$ emissions are asymmetric and tailing toward higher and lower energies, respectively, as shown in the spectrum at $K_{m}=0.70$ $\times 10^{6} \mathrm{~cm}^{-1}$. This is explained as follows. For $K_{m}>k_{0}$, the higher-energy laser is tuned near the transverse-exciton energy, where the band is nearly flat and intermediate excitons are excited with a broad wave number range due to the finite width of the excitation lasers $(\simeq 0.5 \mathrm{meV})$. Correspondingly, biexcitons are also excited in a broad range of $K_{m}$. In addition, $d E_{1} / d K_{m}$, where $E_{1}$ is the final-state polariton energy, increases above $K_{m}=k_{0}$, leading to the broad emissions as observed. This character will be displayed in Fig. 14. Actually, we further tuned both laser energies to study biexcitons with $K_{m}$ more than $\simeq 0.8 \times 10^{6} \mathrm{~cm}^{-1}$, but the features of emission spectra are not different from those at $K_{m}=0.70$ $\times 10^{6} \mathrm{~cm}^{-1}$. 


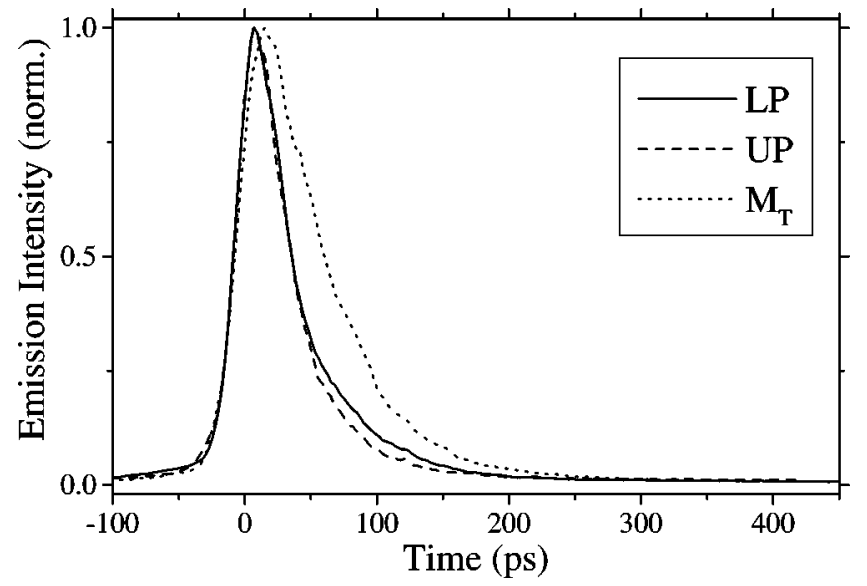

FIG. 6. Time evolution of UP, LP $\left(K_{m}=0\right)$, and $M_{T}$ emissions $\left(K_{m} \simeq 2 k_{0}\right)$.

In Fig. 5, $M_{T}$ and $M_{L}$ emission lines are also observed. They originate from biexciton emissions leaving transverse and longitudinal $Z_{3}-1 s$ excitons behind, respectively. The lines marked EX are from the transverse excitons thus generated. Note that $K_{m} \sim 0$ biexcitons do not decay into such high- $k$ transverse exciton states and the radiative transitions into longitudinal excitons are not allowed for $K_{m}=0$ biexcitons from the polarization-selection rule. Therefore, these emissions come from high- $K_{m}$ biexcitons, which are generated by the internal reflection of the beams in the sample under the collinear excitation condition of $K_{m} \simeq k_{1}^{(0)}+k_{2}^{(0)}$ ( $K_{m}$ is slightly lower than $k_{1}^{(0)}+k_{2}^{(0)}$ because of a finite angle between the input and reflected beams). Sharpness of $M_{T}$ and $M_{L}$ emission lines assures that the weak excitation condition is satisfied. ${ }^{42}$ The emissions marked by LEP and HEP between the two lasers are also from $\simeq k_{1}^{(0)}+k_{2}^{(0)}$ biexcitons. They are observed separately from the excitation lasers because of the finite angle $\left(\simeq 25^{\circ}\right)$ between the excitation beams and the observation direction as shown in Fig. 4. Details of these nearly degenerate two-polariton emissions will be given in Sec. III B.

Figure 6 shows time-resolved UP and LP emissions at $K_{m}=0$ and $M_{T}$ emissions at $K_{m} \simeq 2 k_{0}$ (slightly lower than $2 k_{0}$ ). The time-integrated spectrum for these simultaneously observed lines is given in the bottom of Fig. 5. A single exponential fit yields decay times of 40-45 ps for UP and LP emissions and 50 ps for $M_{T}$ emissions. Since the decay of the $M_{T}$ emission lines reflects the momentum-(phase-) relaxation time rather than the interband-energy-relaxation time, ${ }^{33}$ the radiative lifetime of $\simeq 2 k_{0}$ biexcitons is longer than 50 ps. Actually, the decay time of $M_{L}$ emissions, which is considered to show the true radiative decay, ${ }^{34}$ is about $55-60 \mathrm{ps}$ for the same sample. For $K_{m}=0$ biexcitons, on the other hand, the observed decay time represents the radiative lifetime because there exist no relaxation channels except radiative decay. Thus difference in the radiative lifetimes between $K_{m}=0$ and $K_{m} \simeq 2 k_{0}$ should be larger than in Fig. 6. This reflects different optical decay channels between low- $K_{m}$ and high- $K_{m}$ biexcitons and will be analyzed in Sec. VI.

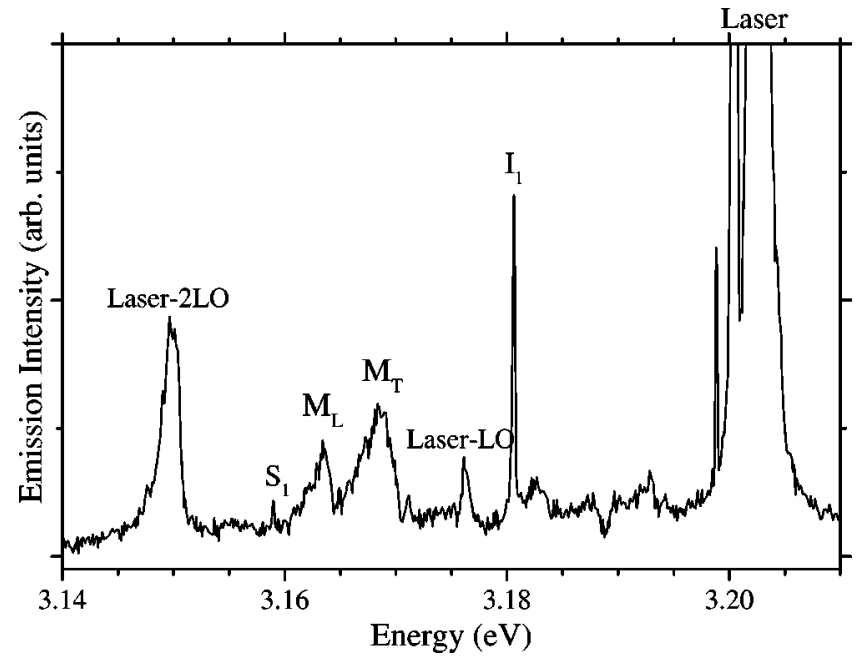

FIG. 7. Emission spectra of $\mathrm{CuCl}$ under resonant excitation of the $Z_{3}$ exciton band. $I_{1}$ and $S_{1}$ lines are from bound excitons, and $M_{T}$ and $M_{L}$ bands are from biexcitons created by exciton mutual collisions.

\section{B. Three-dimensional scattering}

In this section we deal with more complex spectra than in the former section, which arise in the three-demensional scattering condition. Before going to $\mathbf{K}_{m}$-selective excitation, a typical spectrum of collisionally excited biexcitons is shown for comparison.

\section{Collisional excitation}

Figure 7 shows emission spectra at strong excitation of the exciton resonance in $\mathrm{CuCl}$. Beside multiple LO Raman lines and impurity emissions $\left[I_{1}, S_{1}\right.$ (Ref. 49)], doubly peaked, broad emissions ranging from 3.16 to $3.17 \mathrm{eV}$ grow quadratically with the excitation intensity. These are the socalled $M_{T}$ and $M_{L}$ bands, ${ }^{50}$ which are emissions due to biexciton transition to transverse and longitudinal excitons, respectively. Because biexcitons are generated by mutual collisions of excitons, the wave vectors $\left(\mathbf{K}_{m}\right.$ 's) of biexcitons are distributed widely to give rise to broad $M_{T}$ and $M_{L}$ bands. ${ }^{1,42,50}$ The tails of the bands toward the lower-energy side indicate a biexciton distribution toward the higherenergy side (larger $K_{m}$ ). In Fig. 7, no remarkable luminescence features are found around the two-photon degenerate energy, $3.186 \mathrm{eV}$. Minor structures seen around this energy are due to impurity emissions because they are linearly dependent on the excitation intensity. When biexcitons are thermally populated in the $\mathbf{K}_{m}$ space, nearly degenerate twophoton emissions are usually not recognizable ${ }^{50}$ probably because of the lower final polariton density of states around the two-photon degenerate energy than at the exciton resonant energy. ${ }^{1,41}$ This will be clarified in Sec. V A. In order to observe such emissions, $\mathbf{K}_{m}$-selective excitation of biexcitons is necessary as shown in the following.

\section{Degenerate collinear excitation}

Figure 8 shows emission spectra from biexcitons excited by a single beam $\mathbf{k}_{1}^{(0)}$ resonant with degenerate two-photon 


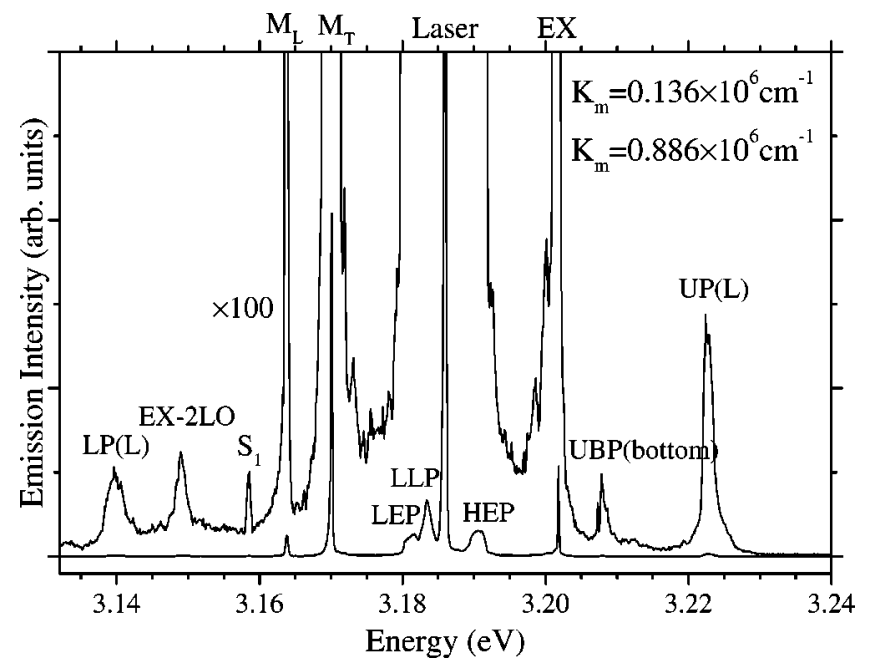

FIG. 8. Emission spectra of biexcitons created by degenerate two-photon excitation with a single beam. The wave numbers of biexcitons simultaneously generated are given at the upper right corner. The HEP, LEP, $M_{T}, M_{L}$, and EX lines are from $\left|\mathbf{K}_{m}\right|$ $=2 k_{0}$ biexcitons, and the $\operatorname{UP}(L), \operatorname{LP}(L)$, and LLP lines are from $\left|\mathbf{K}_{m}\right| \simeq 0$ biexcitons.

energy, $3.186 \mathrm{eV}$. Emission spectra are measured in a backscattering geometry. In this configuration, biexcitons of $\left|\mathbf{K}_{m}\right|=2 k_{0}$ are most efficiently excited from the process of $\mathbf{K}_{m}=\mathbf{k}_{1}^{(0)}+\mathbf{k}_{1}^{(0)}$, but simultaneously $K_{m} \simeq 0$ biexcitons are also generated from the process of $\mathbf{K}_{m} \simeq \pm \mathbf{k}_{1}^{(0)} \mp \mathbf{k}_{1}^{(0)}$ by the reflection of one of the beams on the rear surface to satisfy the antiparallel excitation condition with the other beam. From $2 k_{0}$ biexcitons, $M_{T}$ and $\mathrm{EX}(1 s$ exciton) pair emissions, LEP and HEP pair emissions, and $M_{L}$ emissions are generated. ${ }^{38,42}$ From $K_{m} \simeq 0$ biexcitons, $\operatorname{UP}(L)$ and $\operatorname{LP}(L)$ emissions, which are due to the dissociation into upper- and lower-branch polaritons, ${ }^{38}$ and LLP emissions, which are due to the dissociation into two lower-branch polaritons close to the excitation path, are generated. Previously ${ }^{33,42}$ these lines were not clearly observed in a single spectrum because the excitation laser power was not sufficient.

The emission spectra are not symmetric with respect to $3.186 \mathrm{eV}$ in Fig. 8: The line symmetric to the LLP line is absent, and the sum of the $\operatorname{LP}(L)$ and $\operatorname{UP}(L)$ energies is $6.362 \mathrm{eV}$ in spite of the biexciton energy of $6.372 \mathrm{eV}$. This is because the conjugate polariton pairs to these LLP, $\operatorname{LP}(L)$, and $\operatorname{UP}(L)$ lines are emitted out of the observation solid angle due to the three-dimensional momentum conservation as shown in Fig. 9. The excitation laser beam $(L)$ is at a finite incidence angle while emitted light normal to the surface is collected. As a result, LLP emissions are observed while HLP' $^{\prime}$ emissions are not. The mark ' denotes the emissions out of the observation direction. Conventionally, nearly degenerate pair emissions are called HEP and LEP irrespective of the biexciton wave number. For example, in Ref. 38 the LLP line is referred to as the LEP(II) line. Since the spectra have contributions from both $K_{m} \simeq 0$ and $K_{m} \simeq 2 k_{0}$ biexcitons here, we use HEP and LEP for the emissions from $K_{m}$ $\simeq 2 k_{0}$ biexcitons, and HLP and LLP from $K_{m} \simeq 0$ biexcitons.

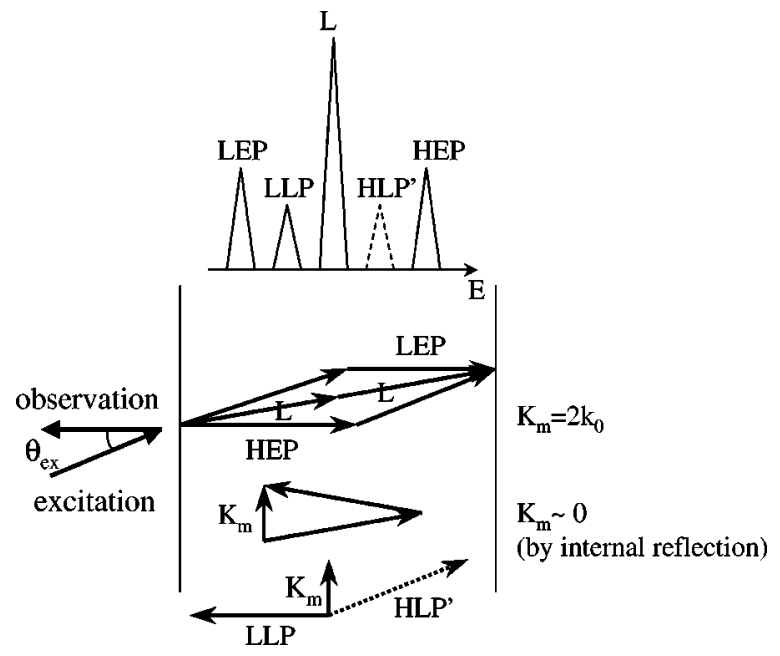

FIG. 9. The schematic of the excitation and emission process for Fig. 8. Together with $\left|\mathbf{K}_{m}\right|=2 k_{0}$ biexcitons, $\left|\mathbf{K}_{m}\right| \simeq\left| \pm \mathbf{k}_{1}^{(0)} \mp \mathbf{k}_{1}^{(0)}\right|$ $\simeq 0$ biexcitons are generated in the direction parallel to the surface by internal reflection of the excitation beam. The LEP and HEP lines from the $2 k_{0}$ biexcitons are observed after internal reflection, and the LLP line from the $K_{m} \sim 0$ biexcitons is directly in the observation direction. The HLP' line, which is the conjugate pair of the LLP, is out of the observation solid angle even after internal reflection.

Similar discussions hold also for the $\operatorname{LP}(L)$ and $\operatorname{UP}(L)$ lines, the conjugate pair emissions $\operatorname{UP}(H)^{\prime}$ and $\operatorname{LP}(H)^{\prime}$ of which are emitted out of the observation direction.

\section{Nondegenerate collinear excitation}

Figure 10 shows emission spectra from biexcitons excited by collinear two-color beams [high-energy laser (HL) and low-energy laser (LL)] in a backscattering geometry. In this case, the processes of $\mathbf{K}_{m}=\mathbf{k}_{1}^{(0)}+\mathbf{k}_{2}^{(0)}$ and $\mathbf{K}_{m} \simeq \pm \mathbf{k}_{1}^{(0)}$ $\mp \mathbf{k}_{2}^{(0)}$ contribute to the biexciton formation. These spectra are also asymmetric as explained in Fig. 11, where LLP and HLP emissions are observed while LLP $^{\prime}$ and HLP' emissions are not. Note that nearly degenerate emissions such as HLP, LLP, HEP, and LEP lines have a larger bandwidth than $M$ and EX lines, reflecting the polariton dispersion curves and the excitation laser bandwidth.

\section{Antiparallel excitation}

Figure 12 shows biexciton emission spectra at an antiparallel excitation configuration. In this case, biexcitons of $\left|\mathbf{K}_{m}\right|=\left|k_{1}^{(0)}-k_{2}^{(0)}\right| \simeq 0$ are predominantly excited, and by internal reflection of the beams $\left|\mathbf{K}_{m}\right| \simeq 2 k_{0}$ biexcitons are also generated as shown in Fig. 13. The emission peaks LEP and HEP between the two lasers are from $\simeq 2 k_{0}$ biexcitons. The bottom figure shows the emissions exactly from $\left|\mathbf{K}_{m}\right|=2 k_{0}$ biexcitons, i.e., completely degenerate two-photon emissions. They evolve from broad bands into sharp peaks from bottom to top with increasing $K_{m}$. As explained in Sec. IV B, this feature reflects the finite laser bandwidth and po- 


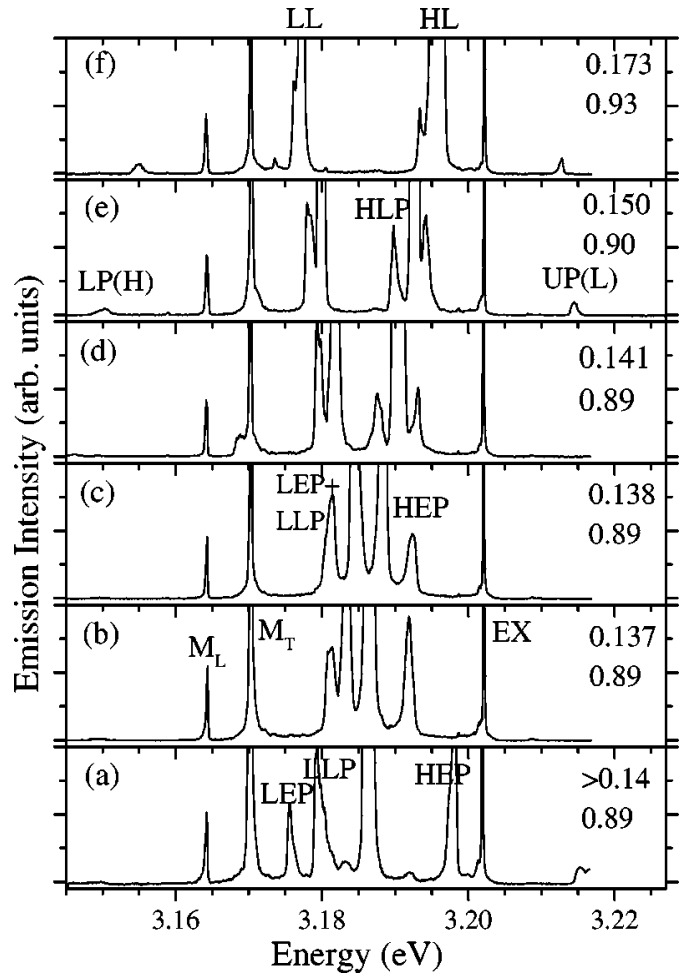

FIG. 10. Emission spectra of biexcitons created by nondegenerate two-photon excitation with collinear two-color beams. The wave numbers of biexcitons simultaneously created are given at the upper right corner in units of $10^{6} \mathrm{~cm}^{-1}$. The spectrum (a) is at a different excitation/observation angle from the spectra (b)-(f). The HEP, LEP, $M_{T}, M_{L}$, and EX lines are from $\left|\mathbf{K}_{m}\right|=k_{1}^{(0)}+k_{2}^{(0)}$ biexcitons, and the $\operatorname{UP}(L), \operatorname{LP}(H), \mathrm{HLP}$, and LLP lines are from $\left|\mathbf{K}_{m}\right| \simeq 0$ biexcitons. HEP, LEP, and EX are emissions reflected on the rear surface, and $\operatorname{UP}(L), \operatorname{LP}(H), \operatorname{HLP}, \mathrm{LLP}$, and $M_{T}$ are in the observing direction.

lariton dispersion curves, and the broadest structure at the bottom is due to the singularity of JDPS. ${ }^{44}$ The minor structures seen around 3.186-3.187 eV are due to impurity emissions because they are present at single-beam excitation with the high-energy laser HL.

It is much more difficult to observe completely degenerate emissions from $K_{m}=0$ biexcitons, because in order to avoid overlap with pump scattering, the LBP-UBP excitation path should be used, where the generation efficiency of biexcitons is considerably reduced as shown in Sec. V B.

\section{DETERMINATION OF POLARITON DISPERSION}

In this section, using the one-dimensional scattering spectra in Fig. 5, the polariton dispersion is precisely determined. Then, with this dispersion, the emission peaks of the threedimensional scattering spectra in Figs. 8, 10, and 12 are precisely assigned.

\section{A. One-dimensional scattering}

The observed energies of the UP and LP emissions in Fig. 5 reflect polariton dispersion curves in $\mathrm{CuCl}$. The standard one-oscillator polariton dispersion curves ${ }^{21}$ predict $3.283 \mathrm{eV}$

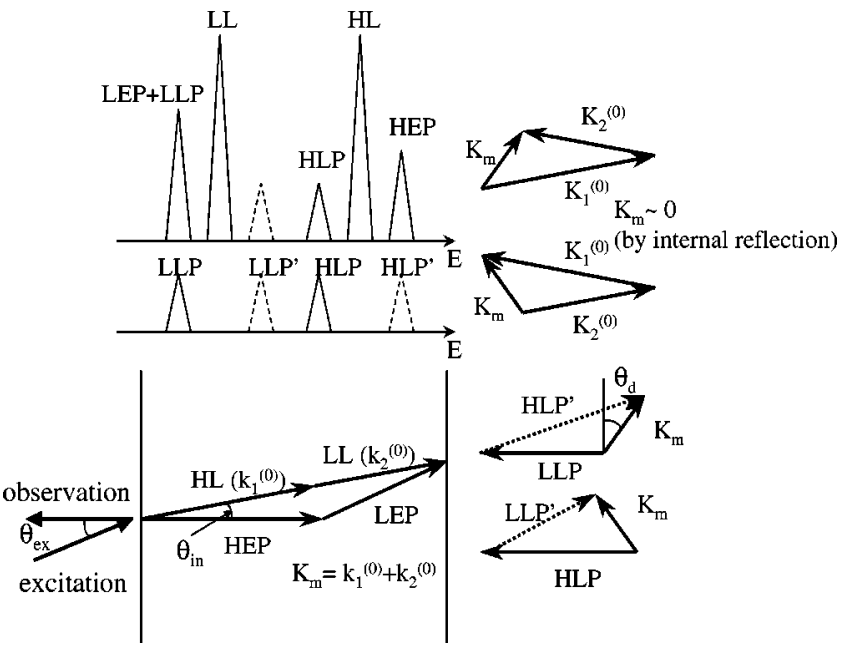

FIG. 11. The schematic of the excitation and emission process for Fig. 10. Together with $\left|\mathbf{K}_{m}\right|=k_{1}^{(0)}+k_{2}^{(0)}$ biexcitons, $\left|\mathbf{K}_{m}\right|$ $\simeq\left| \pm \mathbf{k}_{1}^{(0)} \mp \mathbf{k}_{2}^{(0)}\right| \simeq 0$ biexcitons are generated at the angle $\theta_{d}$ from the surface by internal reflection of the excitation beams. The LLP and HLP lines, which are due to emissions at $90^{\circ} \pm \theta_{d}$ from the wave vectors of the $K_{m} \simeq 0$ biexcitons, are in the observation direction while the $\mathrm{HLP}^{\prime}$ and LLP' lines, which are the conjugate pairs of the LLP and HLP, are out of the observation solid angle. The energies of LEP and LLP (HEP and HLP') lines almost coincide with each other, thus the two lines overlap to show the higher peak intensity than the HEP lines. A double-peak structure of the LEP lines is recognized by a closer look of the lines in Fig. 10.

and $3.089 \mathrm{eV}$ for the UP and LP emissions from $K_{m}=0$ biexcitons, respectively. These energies deviate far from the observed UP and LP energies, thus requiring for higher oscillators to be included in the dispersion curves. Need for such a multiple oscillator model was previously discussed in $\mathrm{CuCl},{ }^{38,51,52}$ but the model was compared with the experiments only in narrow energy ranges of observation. The wide energy range of the present observation allows us to determine multiple polariton dispersion curves more precisely.

In order to explain the emission energies from low- $K_{m}$ biexcitons, the peak energies of the two excitation lasers and four emission lines are plotted against $K_{m}$ in Fig. 14. The experimental results are obtained from two samples investigated in detail. Polariton dispersion curves are calculated by a two-oscillator model ( $Z_{3}$ and $Z_{1,2} 1$ s exciton $)$ as follows: ${ }^{38}$

$$
\begin{aligned}
\frac{\hbar c^{2} k^{2}}{E^{2}} & =\varepsilon_{b}+\frac{\alpha_{1 s} E_{T}^{2}}{E_{T}^{2}-E^{2}}+\frac{\alpha_{Z} E_{t}^{2}}{E_{t}^{2}-E^{2}} \\
& =\varepsilon_{b} \frac{\left(E_{L}^{2}-E^{2}\right)\left(E_{l}^{2}-E^{2}\right)}{\left(E_{T}^{2}-E^{2}\right)\left(E_{t}^{2}-E^{2}\right)} .
\end{aligned}
$$

Here $E_{T}\left(E_{L}\right)$ and $E_{t}\left(E_{l}\right)$ are the transverse (longitudinal) $Z_{3}$ and $Z_{1,2}$ exciton energies, respectively $\left(E_{T} \equiv E_{1 s}, E_{t}\right.$ $\equiv E_{Z}$ ), and $\alpha_{1 s}$ and $\alpha_{Z}$ are exciton-photon coupling constants. For one-oscillator polaritons, $E_{L}^{\prime}=\sqrt{1+\alpha_{1 s} / \varepsilon_{b}} E_{T}$ and $E_{l}^{\prime}=\sqrt{1+\alpha_{Z} / \varepsilon_{b}} E_{t}$ are the longitudinal energies, but in 


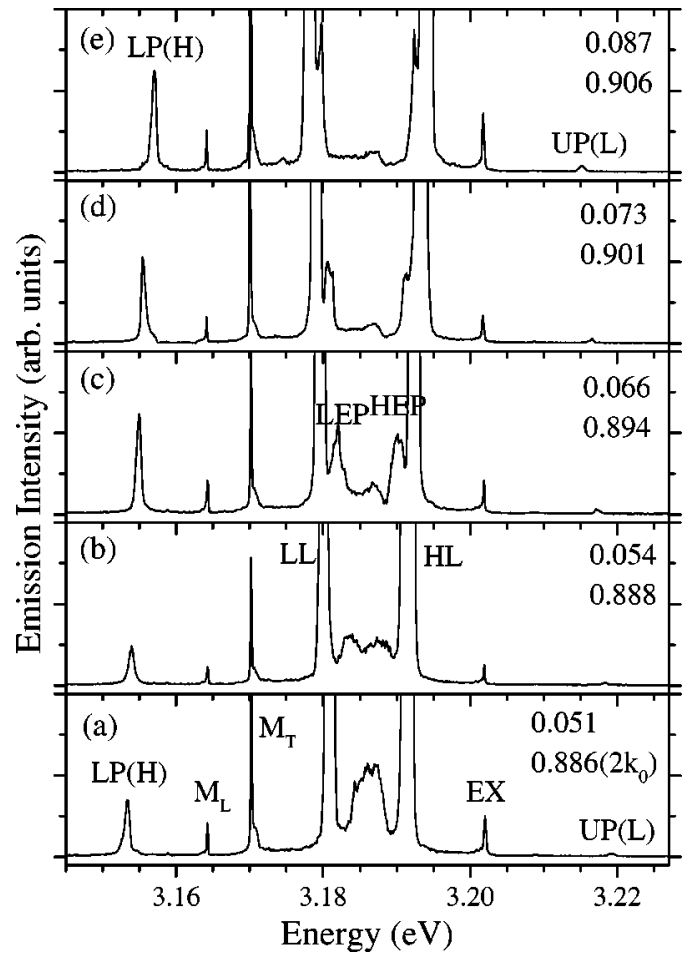

FIG. 12. Emission spectra of biexcitons created by nondegenerate two-photon excitation with antiparallel beams. The wave numbers of biexcitons simultaneously created are given at the upper right corner in units of $10^{6} \mathrm{~cm}^{-1}$. The HEP, LEP, $M_{T}, M_{L}$, and EX lines are from $\left|\mathbf{K}_{m}\right| \simeq 2 k_{0}$ biexcitons, and the $\operatorname{UP}(L)$ and $\operatorname{LP}(H)$ lines are from $\left|\mathbf{K}_{m}\right|=\left|k_{1}^{(0)}-k_{2}^{(0)}\right|$ biexcitons.

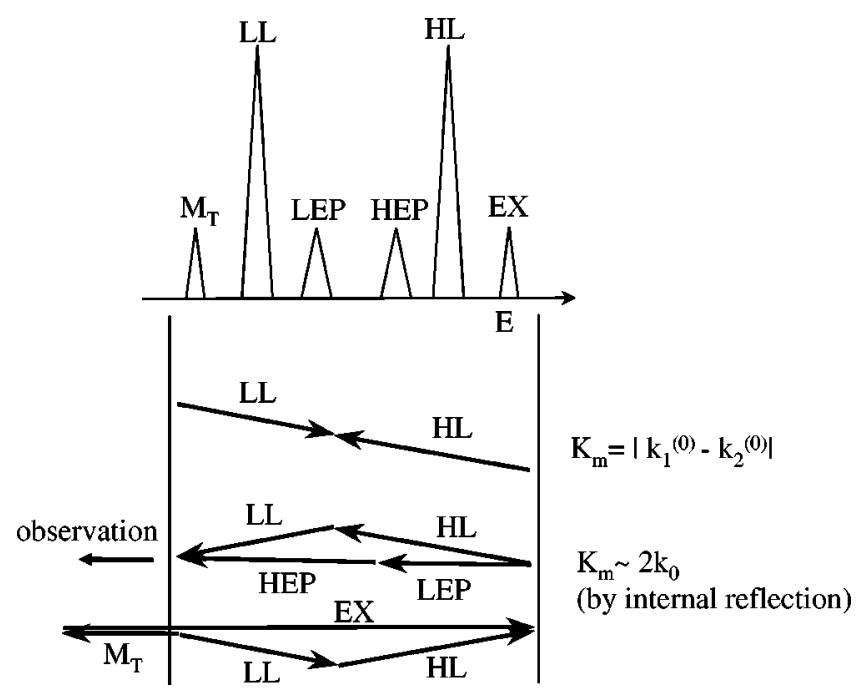

FIG. 13. The schematic of the excitation and emission process for Fig. 12. Together with $\left|\mathbf{K}_{m}\right|=\left|k_{1}^{(0)}-k_{2}^{(0)}\right| \simeq 0$ biexcitons, $\left|\mathbf{K}_{m}\right|$ $\simeq\left| \pm \mathbf{k}_{1}^{(0)} \mp \mathbf{k}_{2}^{(0)}\right| \simeq 2 k_{0}$ biexcitons are generated in the direction almost normal to the surface by internal reflection of the excitation beams to emit HEP, LEP, $M_{T}$, and EX emissions. For the calculations shown in Figs. 15 and 16, both wave vectors of $\left|\mathbf{K}_{m}\right|=\mid k_{1}^{(0)}$ $-k_{2}^{(0)} \mid$ and $\left|\mathbf{K}_{m}\right| \simeq 2 k_{0}$ biexcitons can be taken to be normal to the surface without appreciable errors.

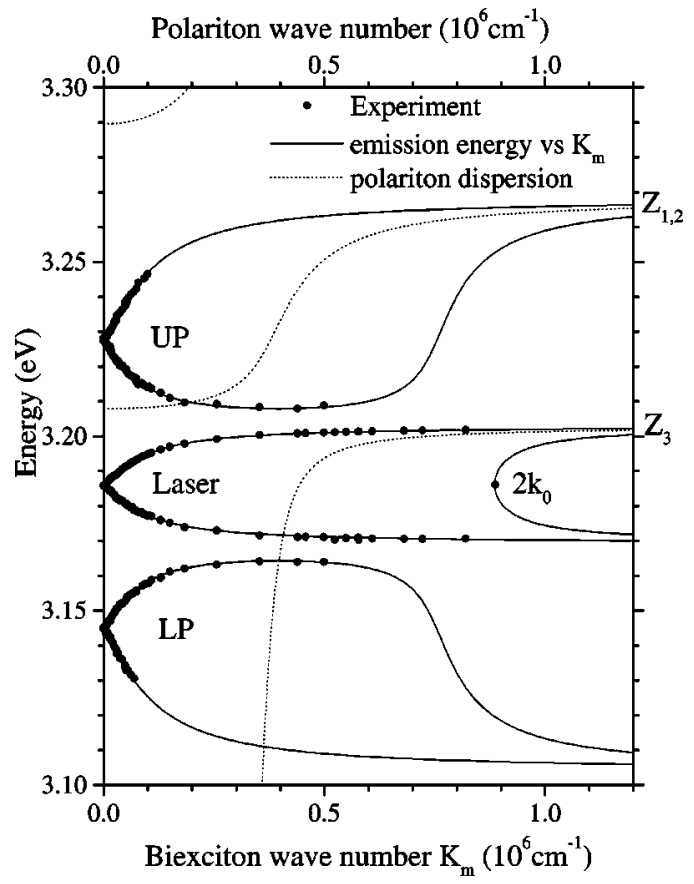

FIG. 14. One-dimensional scattering: The two excitation laser energies and four emission energies as a function of the biexciton wave number $K_{m}$ are displayed with experimental results (dots) in Fig. 5, calculated results (solid curves) by a two-oscillator $\left(Z_{3}\right.$ and $Z_{1,2}$ ) model, and the corresponding polariton dispersion curves (dashed curves). The gradients of the curves, $d E / d K_{m}$, explain broad features of the UP and LP lines at $K_{m}=0.70 \times 10^{6} \mathrm{~cm}^{-1}$ in Fig. 5 (see text in Sec. III). Since the UP and LP lines show the similar broad features above $K_{m}=0.5 \times 10^{6} \mathrm{~cm}^{-1}$, the experimental points are not reliably obtained in this region.

Eq. (2), $E_{L}<E_{L}^{\prime}$ and $E_{l}>E_{l}^{\prime}$ because of the polariton coupling of the two oscillators (Appendix A).

The energies of emitted polaritons as a function of $K_{m}$ are calculated under energy and momentum conservation to fit the experimental results, with $E_{t}, E_{l}^{\prime}$, and $\varepsilon_{b}$ as variable parameters. The biexciton wave number in the experiment is determined by $K_{m}=k_{1}^{(0)}-k_{2}^{(0)}$, where the input polariton wave numbers of $k_{1}^{(0)}$ and $k_{2}^{(0)}$ are iteratively calculated from the dispersion curves assumed with the given pump-laser energies. The solid curves in Fig. 14 give the best fit, showing an excellent agreement between the theory and experiment. The calculated UP and LP energies are $3.228 \mathrm{eV}$ and $3.144 \mathrm{eV}$, respectively. Here we employed a quasiparticle excitation scheme by Tait, ${ }^{53}$ where the wave number is well defined as a real value and the frequency is a complex value such that excited particles decay as a function of time. The quasiparticle solution of polariton dispersions is insensitive to damping parameters in the frequency. In this case the damping of the $Z_{1,2}$ exciton can be set to be zero without losing reliability of the fitting, although it is expected to be around $10 \mathrm{meV} .{ }^{54}$ The good fit indicates that a quasiparticle picture is adequate for describing the radiative decay of twophoton generated biexcitons.

Parameter values determined from the fitting are listed in Table I. ${ }^{45}$ For the $Z_{3}$ exciton and biexciton, the values in Ref. 21 are used (the $Z_{1,2}$ exciton mass is taken to be the same as 
TABLE I. Parameters used for fitting to the experimental results. The energy unit is eV. $E_{L}^{\prime}=\left(1+\alpha_{1 s} / \varepsilon_{b}\right)^{1 / 2} E_{T}$ and $E_{l}^{\prime}=(1$ $\left.+\alpha_{Z} / \varepsilon_{b}\right)^{1 / 2} E_{t}$.

\begin{tabular}{cccccccc}
\hline \hline Parameters & $\varepsilon_{b}$ & $E_{T}$ & $E_{L}^{\prime}$ & $E_{L}$ & $E_{t}$ & $E_{l}^{\prime}$ & $E_{l}$ \\
\hline & 4.3 & 3.2022 & 3.21 & 3.2079 & 3.267 & 3.288 & 3.2896 \\
\hline \hline
\end{tabular}

the $Z_{3}$ exciton) and $\varepsilon_{b}$ is so determined that $2 k_{0}=0.89$ $\times 10^{6} \mathrm{~cm}^{-1}$ (Ref. 55). The $Z_{1,2}$ exciton parameters and $\varepsilon_{b}$ here coincide well with the values in Ref. 56. The dispersion curves determined shows that the polariton above $3.228 \mathrm{eV}$ (the UP energy for the $K_{m}=0$ biexciton) is better described by the $Z_{1,2}-1 s$ LBP rather than by the $Z_{3}-1 s$ UBP. This nature is clarified by comparing the magnitudes of the $Z_{1,2}$ and $Z_{3}$ exciton components in the polariton [see Eq. (13)]. Details of $Z_{1,2}$ exciton-polaritons will be reported in Ref. 48.

\section{B. Three-dimensional scattering}

As seen in Figs. 8 and 10, the LLP and HLP emissions have a much larger intensity than the $\operatorname{UP}(L)$ and $\operatorname{LP}(H, L)$ emissions. The biexciton radiative decay theory on the BP model will be tested by a precise comparison of these emission intensities with the theory. For this purpose, we should compare emissions from biexcitons with the same wave vector and emitted in the same direction. In this section, we make a precise assignment of all the observed emission lines under the three-dimensional energy-momentum conservation law, in preparation for the analysis on the emission intensity in Sec. V.

\section{Collinear excitation}

The assignment of the emission processes schematically shown in Figs. 9 and 11 for the collinear excitation case is confirmed by the following procedure. We have calculated the emission energy as a function of $K_{m}$ at fixed incidence and observation angles using the polariton dispersion determined in Sec. IV A. Given the two laser energies $E_{1}^{(0)}$ and $E_{2}^{(0)}$ and the external incident angle $\theta_{\text {ex }}$, the wave vectors of low- $K_{m}$ biexcitons and the energies of polariton emissions normal to the surface, i.e., LLP, $\operatorname{HLP}, \operatorname{UP}(L, H)$, and $\mathrm{LP}(L, H)$ emissions are calculated as follows.

For the external incidence angle $\theta_{e x}$, the internal incidence angle $\theta_{i n}$ is obtained from

$$
\sin \theta_{e x}=n(E) \sin \theta_{i n} .
$$

Here, the refractive index $n(E)$ is taken to be a constant, the value at $3.186 \mathrm{eV}$. Then, for the polariton wave numbers $k_{1}^{(0)}=k\left(E_{1}^{(0)}\right)$ and $k_{2}^{(0)}=k\left(E_{2}^{(0)}\right)$, low- $K_{m}$ biexcitons of

$$
K_{m}=\sqrt{\left(k_{1}^{(0)}\right)^{2}+\left(k_{2}^{(0)}\right)^{2}-2 k_{1}^{(0)} k_{2}^{(0)} \cos \left(2 \theta_{i n}\right)}
$$

are generated by internal reflection. The deviation angle $\theta_{d}$ of $\mathbf{K}_{m}$ from the sample surface, as shown in Fig. 11, is obtained from

$$
K_{m} \cos \theta_{d}=\left(k_{1}^{(0)}+k_{2}^{(0)}\right) \sin \theta_{i n} .
$$

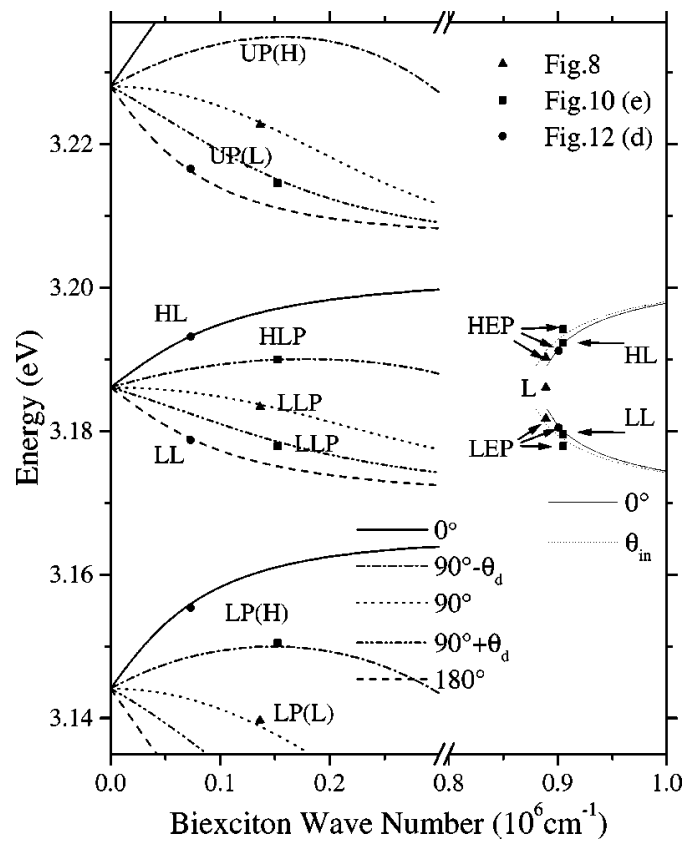

FIG. 15. Three-dimensional scattering: The energies of polariton emissions from biexcitons as a function of $K_{m}$ calculated at the laser external incidence angle $\theta_{e x}=25^{\circ}$ and at the normal observation angle. The calculated curves are labeled by the internal polariton scattering angle $\theta_{o b s}$ from $\mathbf{K}_{m}$. The curves for the conjugated pair emissions $\left(H L P^{\prime}, L L P^{\prime}, U P^{\prime}, L P^{\prime}\right)$, which are symmetric to the curves labeled $\theta_{\text {obs }}=90^{\circ}, 90^{\circ} \pm \theta_{d}\left(\theta_{d}=23^{\circ}\right)$ with respect to 3.186 $\mathrm{eV}$, are not displayed because they are out of the observation direction. Triangles $(\boldsymbol{\Delta})$ shown experimental results $[L L P, L P(L), U P(L)$, $L E P, H E P$ emissions and the excitation laser $L]$ for the degenerate excitation case in Fig. 8. Squares (ם) show experimental results $[L L P, H L P, L P(H), \operatorname{UP}(L), L E P, H E P, L L, H L]$ for the nondegenerate excitation case in Fig. 10(e). Note that $\theta_{d}=15^{\circ}$ for the spectra in Figs. 10 (d) and $\theta_{d}=34^{\circ}$ in Fig. 10(f), as the direction of $\mathbf{K}_{m}$ is varied depending on the excitation laser energies. Circles $(\mathbf{O})$ show experimental results $[L E P, H E P, L P(H), U P(L), L L, H L]$ for the antiparallel excitation case in Fig. 12(d).

Thus, polaritons emitted in the direction normal to the sample surface is at the angle $\theta_{o b s}$ from $\mathbf{K}_{m}$,

$$
\theta_{o b s}=90^{\circ} \pm \theta_{d}
$$

When the two laser energies are degenerate $\left(k_{1}^{(0)}=k_{2}^{(0)}\right)$, the wave vectors of low- $K_{m}$ biexcitons are parallel to the surface and polariton emissions at the angle $\theta_{o b s}=90^{\circ}$ are observed $\left(\theta_{d}=0^{\circ}\right)$ as shown in Fig. 9. Figure 15 shows the calculated emission energy as a function of biexciton wave number at the angles $\theta_{o b s}$ of $0^{\circ}, 180^{\circ}, 90^{\circ}$, and $90^{\circ} \pm \theta_{d}$ with the external (experimental) incidence angle $\theta_{e x}=25^{\circ}$. The curves denoted by $90^{\circ}$ and by $90^{\circ} \pm \theta_{d}\left(\theta_{d}=23^{\circ}\right)$ explain the spectra in Figs. 8 and 10(e), respectively. The wave vectors of high- $K_{m}$ biexcitons simultaneously generated are given by $K_{m}=k_{1}^{(0)}+k_{2}^{(0)}$, and the energies of HEP and LEP emissions normal to the surface $\left(\theta_{o b s}=\theta_{i n}\right)$ are also displayed in Fig. 15. The experimental points given by the filled triangles and squares agree very well with the calculated curves. 


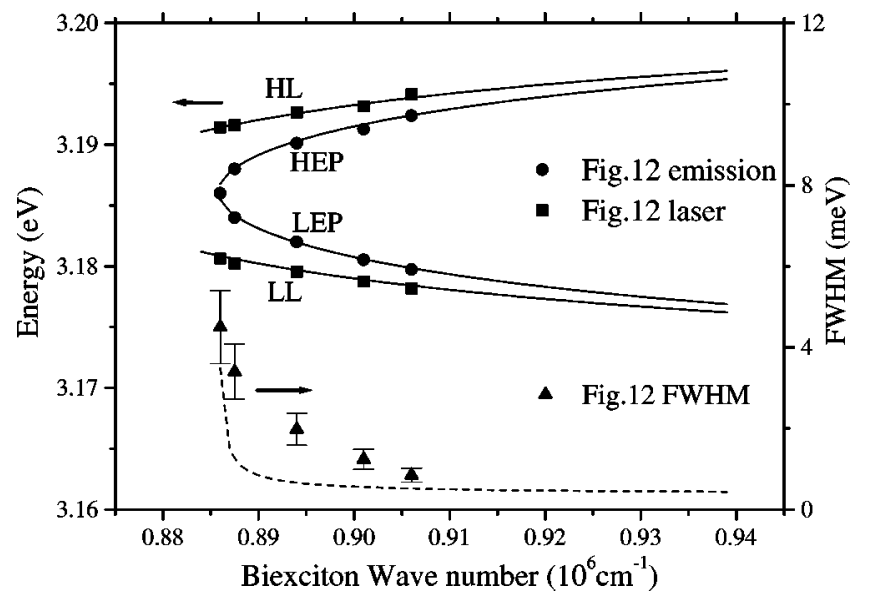

FIG. 16. The energies of the HEP and LEP emission lines (circles -), the energies of the excitation lasers HL and LL (squares $\mathbf{0}$ ), the full widths at half maximum of the HEP and LEP lines (triangles $\boldsymbol{\Delta}$ ) taken from Fig. 12, and the calculated emission energy (solid curves) and width (dashed curve) at the incidence angle $\theta_{e x}=30^{\circ}$ of the lasers with the bandwidths of $0.5 \mathrm{meV}$.

From Fig. 15, it is confirmed that the LLP, $\operatorname{LP}(L)$, and $\mathrm{UP}(L)$ lines in Fig. 8 and the HLP and LP $(H)$ lines in Fig. 10 come from polaritons emitted in the same direction and from biexcitons of the same wave vector.

\section{Antiparallel excitation}

The observed emission energies for the antiparallel excitation case in Fig. 12 are explained by the calculation as follows. For the internal incidence angle $\theta_{i n}$, the wave number of high- $K_{m}$ biexcitons is obtained by

$$
K_{m}=\sqrt{\left(k_{1}^{(0)}+k_{2}^{(0)}\right)^{2} \cos ^{2} \theta_{i n}+\left(k_{1}^{(0)}-k_{2}^{(0)}\right)^{2} \sin ^{2} \theta_{i n}} .
$$

Since the wave vector is almost normal to the surface, we can readily obtain the energies of HEP and LEP emissions at the exit angle of $\theta_{\text {out }}=0^{\circ}$. Further, we can estimate the widths of emission lines from the excitation laser bandwidth, $\simeq 0.5 \mathrm{meV}$, and the polariton dispersion curves as follows. For $K_{m}=k_{1}+k_{2}$ and $\omega_{m}=\omega_{1}+\omega_{2}$, the relation $\Delta K_{m}=\Delta k_{1}$ $+\Delta k_{2}=\left(d k_{1} / d \omega_{1}-d k_{2} / d \omega_{2}\right) \Delta \omega_{1}$ holds. If Gaussian shapes are assumed for the two excitation pulses with the same widths $\Delta \omega_{0}$, the emission line width $\Delta \omega$ is calculated as

$$
\begin{gathered}
\Delta K_{m}^{(0)}=\left(\frac{d k_{1}^{(0)}}{d \omega_{1}}-\frac{d k_{2}^{(0)}}{d \omega_{2}}\right) \frac{\Delta \omega_{0}}{\sqrt{2}}, \\
\Delta \omega=\frac{\Delta K_{m}^{(0)}}{\left(\frac{d k_{1}}{d \omega_{1}}-\frac{d k_{2}}{d \omega_{2}}\right)} .
\end{gathered}
$$

Here, the factor $1 / \sqrt{2}$ is needed in the absorption process because the molecule distribution is proportional to the product of two Gaussian beams $I_{1}(\omega) I_{2}(\omega)$. Figure 16 shows the results of calculation in reasonable agreement with the experimental widths. The observed width is larger than the cal- culated width, partly because of the contribution from the finite solid angle of observation, which is estimated to be about $0.8 \mathrm{meV}$ (FWHM for $\pm 2.5^{\circ}$ ), and partly because of the elastic scattering of high- $K_{m}$ biexcitons, ${ }^{33}$ which obscures an initially sharp distribution of the biexciton wave vector to cause an additional broadening of the emission width.

We can compare the intensity ratio between $M_{T}$ and LEP, HEP lines if high- $K_{m}$ biexcitons are generated evenly in both directions by internal reflection. This condition is considered to be satisfied because there is no reason for asymmetry. If not, they cannot be compared as they are since the $M_{T}$ emission is in the backward direction and the LEP and HEP emissions are in the forward direction.

\section{ANALYSIS ON BIEXCITON EMISSION INTENSITY}

\section{A. Comparison with biexciton radiative decay theory}

The strength of polariton effects is scaled by the polariton parameter $\Omega_{n}{ }^{2,31}$ defined by $\hbar \Omega_{n}=\left(\alpha_{n} / \varepsilon_{b}\right)^{1 / 2} E_{n s}$ $\simeq \sqrt{2 \Delta_{L T}^{(n)} E_{n s}}$, where $E_{n s}$ is the $n$th exciton energy and $\Delta_{L T}^{(n)}$ is its longitudinal-transverse splitting. This gives the polariton Rabi frequency, i.e., the splitting energy at the intersection of photon and exciton branches $\hbar k=\sqrt{\varepsilon_{b}} E_{1 s} / c$. Since $\Omega_{n}$ for the ground-state exciton in $\mathrm{CuCl}$ is larger $\left(\hbar \Omega_{1 s}\right.$ $\sim 224 \mathrm{meV})$ than the biexciton binding energy $\left(\epsilon^{m}=32\right.$ $\mathrm{meV}$ ), a perturbative treatment on the exciton-photon interaction is not appropriate but the BP model $^{19}$ should be applied for the biexciton optical decay in $\mathrm{CuCl}$ especially for a low- $K_{m}$ region. This is usually the case for other bulk directgap semiconductors too. In this model, the biexciton resonant dissociation into two polariton eigenstates is rigorously treated and is interpreted to be mediated by the excitonexciton attractive Coulomb interaction. The radiative transition probability of biexcitons of $\left(E_{m}, \mathbf{K}_{m}\right)$ into polaritons of $\left(E_{1}, \mathbf{k}_{1}\right)$ and $\left(E_{2}, \mathbf{k}_{2}\right)$ is expressed by ${ }^{39,40}$ (see Appendix B)

$$
\begin{aligned}
\gamma\left(\mathbf{K}_{m}, \mathbf{k}_{1}\right) \propto & \rho\left(\mathbf{k}_{1}, \mathbf{k}_{2}\right)\left|\mathbf{e}_{1} \cdot \mathbf{e}_{2}\right|^{2} \\
& \times\left|B_{1 s}\left(k_{1}\right) B_{1 s}\left(k_{2}\right) \epsilon^{m} \int d \mathbf{R} \Psi(\mathbf{R})\right|^{2},
\end{aligned}
$$

where $\rho\left(\mathbf{k}_{1}, \mathbf{k}_{2}\right)$ is the JDPS given by

$$
\rho\left(\mathbf{k}_{1}, \mathbf{k}_{2}\right)=\frac{1}{\frac{d E_{1}}{d k_{1}}-\frac{d E_{2}}{d k_{2}} \cos \left(\theta_{1}+\theta_{2}\right)}
$$

with the internal scattering angle $\theta\left(\theta=\theta_{1}+\theta_{2}\right)$ between $\mathbf{k}_{1}$ and $\mathbf{k}_{2}, \theta_{1,2}$ is the angle between $\mathbf{k}_{1,2}$ and $\mathbf{K}_{m},\left|\mathbf{e}_{1} \cdot \mathbf{e}_{2}\right|^{2}$ is the polarization factor given by $1+\cos ^{2} \theta,{ }^{17} B_{1 s}$ is the Hopfield coefficient that represents the $1 \mathrm{~s}$ exciton component in the polariton, ${ }^{2,17,32} \epsilon^{m}$ is the biexciton binding energy, and $\Psi(\mathbf{R})$ is the biexciton envelope wave function. Note that $\theta_{1}$ $=\theta_{o b s}$ in Sec. IV. Equation (10) is a special case of Eq. (3) in Ref. 39: Only $C_{1,1}(0)=\int d \mathbf{R} \Psi(\mathbf{R})$ is taken into account with the approximate biexciton wave function of $\Psi(\mathbf{R}) \phi_{1 s}\left(\mathbf{r}_{1}\right) \phi_{1 s}\left(\mathbf{r}_{2}\right)$, where $\phi_{1 s}$ is the envelope wave function of the $Z_{3}-1 s$ exciton, and $K_{m}$ and $k_{1}$ are taken to be zero 
$\left[C_{1,1}(k)=C_{1,1}(0), \epsilon^{m}(k)=\epsilon^{m}(0)\right]$ because they are much smaller than the inverse radius of the biexciton wave function. ${ }^{3,4}$ The biexciton translational motion, $K_{m}$, affects the radiative decay rate for the much higher- $K_{m}$ region than the present region of observation as shown in Fig. 5 of Ref. 33. The polarization factor, which comes from the spin structures of excitons, has not been included previously ${ }^{39,40}$ because it is not needed for the analysis on the emission processes in the one-dimensional scattering geometry.

The biexciton emission intensity is obtained as

$$
I\left(\mathbf{K}_{m}, E_{1}\right) \propto T\left(E_{1}\right) k_{1}^{2} \gamma\left(\mathbf{K}_{m}, \mathbf{k}_{1}\right),
$$

where the factor of $k_{1}^{2}$ comes from the solid angle of observation and $T\left(E_{1}\right)$ is the transmissivity of the sample given by $4 n\left(E_{1}\right) /\left[1+n\left(E_{1}\right)\right]^{2}$. For the calculation, the twooscillator polariton dispersion determined in Fig. 14 is used and $B_{1 s}$ is evaluated by the Hopfield theory generalized to multiple-branch polaritons ${ }^{32,57}$ such that the modification by the $Z_{1,2}$ exciton is included. The explicit form of $B_{1 s}$ including the $Z_{1,2}$ exciton reads

$$
\begin{aligned}
B_{1 s}(k)= & i\left(\frac{\alpha_{1 s}}{\varepsilon_{b}}\right)^{1 / 2} \frac{X_{1 s}^{1 / 2}}{1-X_{1 s}^{2}} \\
& \times \frac{1}{\left[1+\frac{\alpha_{1 s}}{\varepsilon_{b}}\left(1-X_{1 s}^{2}\right)^{-2}+\frac{\alpha_{Z}}{\varepsilon_{b}}\left(1-X_{Z}^{2}\right)^{-2}\right]^{1 / 2}},
\end{aligned}
$$

where $X_{1 s}=E(k) / E_{1 s}$ and $X_{Z}=E(k) / E_{Z}$. This form is reduced to the well-known form for the one-oscillator case $^{2,17,18}$ in the limit of $\alpha_{Z}=0$.

\section{1. $K_{m}$-selective excitation}

Figure 17 shows the respective contributions of the factors in Eq. (12) to the emission intensity, calculated at the fixed scattering angle of $\theta=\pi$ with the biexciton wave number varied around $K_{m} \sim 0$. The correction by the $k_{1}^{2}$ factor comes from the mode density within a fixed, finite solid angle of observation in the experiment. Here, we do not consider damping of polaritons during the propagation in a sample, which should cause a significant decrease in the emitted photon intensity as the energy approaches the exciton energy, $3.202 \mathrm{eV}^{58,59} \mathrm{~A}$ few percent correction of the emission intensity by the photon energy is also neglected. As shown by the thick solid curves in Fig. 17(a), without the correction factor, the intensity $\left(\gamma \propto \rho|B B|^{2}\right)$ is largest at the degenerate two-polariton emission energy $3.186 \mathrm{eV}$ and shows wide resonance behavior reflecting the large-polariton Rabi splitting $\hbar \Omega_{1 s}$. This is in remarked contrast with the two-photon resonant absorption intensity, which shows a sharp resonance enhancement at the exciton energy. ${ }^{31}$

Figure 18 shows the respective contributions in Eq. (12) to the emission intensity, calculated at the fixed biexciton wave number of $\left|\mathbf{K}_{m}\right|=2 k_{0}$ with the scattering angle $\theta$ varied. The matrix element (thick solid curve) shown in Fig. 18(a) is the same as that in Fig. 17(a), and the JDPS (thin solid curves) depends on the internal scattering angle $\theta$. The correction factor by the transmission and solid angle of ob-
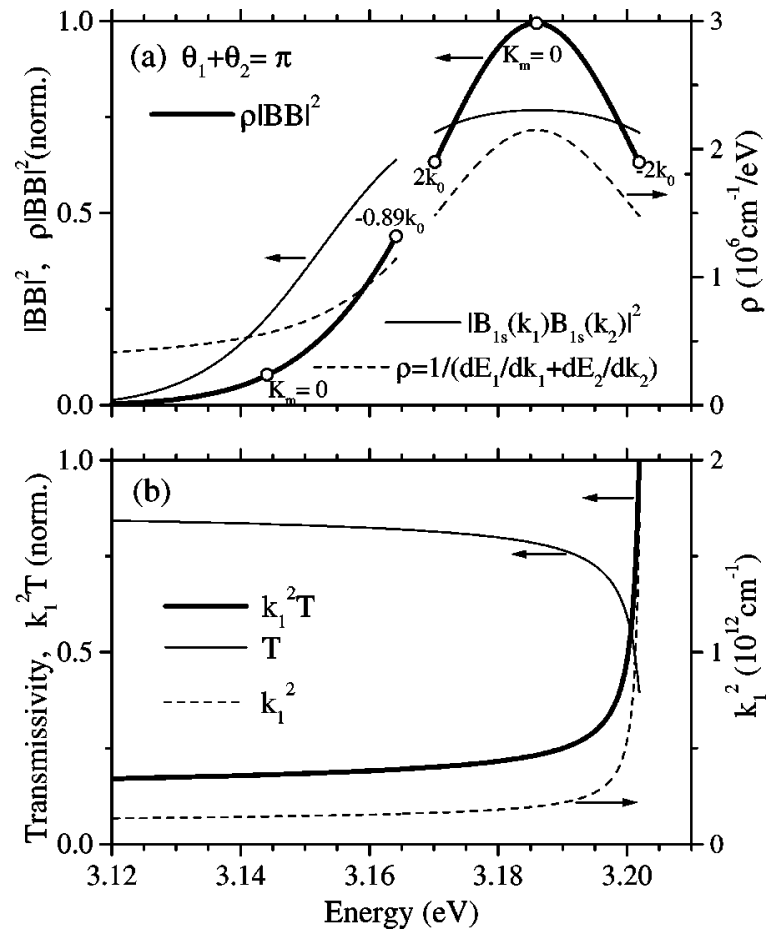

FIG. 17. (a) The biexciton radiative transition probability as a function of the emitted polariton energy (thick solid curves) on the antiparallel emission condition of $\theta_{1}+\theta_{2}=\pi$ with $K_{m}$ varied from $-2 k_{0}$ to $2 k_{0}$ for LBP-LBP and from $-0.89 k_{0}$ to $2 k_{0}$ for UBPLBP, due to the transition matrix element $\left|B_{1 s}\left(k_{1}\right) B_{1 s}\left(k_{2}\right)\right|^{2}$ (thin solid curves) and the joint density of polariton states $\rho$ $=1 /\left(d E_{1} / d k_{1}+d E_{2} / d k_{2}\right)$ (dashed curves). (b) The correction factor (thick solid curve) for the detected emission intensity due to the sample transmission, $T(E)$ (thin solid curve), and due to the solid angle of observation, $k_{1}^{2}$ (dashed curve).

servation is the same as in Fig. 17(b). Note that the matrix element $|B B|^{2}$ is almost constant in the energy range of $3.17-3.202 \mathrm{eV}$. This means that it is practically impossible to identify the contribution of the Hopfield coefficients $(|B B|$ formula) as long as the observation is limited to the LBPLBP channels as has been done previously.

The results of calculation with all the contributions together in Eq. (12) are shown by the solid curves in Fig. 19 in comparison with the experimental results. Note that there are no free fitting parameters used for the calculation, and that the curves close to $3.202 \mathrm{eV}$ cannot be directly compared with the experimental results since the damping is omitted. The filled circles in Fig. 19(a) shows the intensity ratios of the LLP and $\operatorname{LP}(L)$ emissions and of the HLP and $\operatorname{LP}(H)$ emissions in Figs. 8 and 10, taken from the results on four samples. The open triangles and filled circles in Fig. 19(b) show the intensity ratios of the HEP, LEP, and $M_{T}$ lines in Figs. 10(a), 10(b), and 12. As proved in Sec. IV, these emission lines are from biexcitons with the same wave vector and emitted in the same direction. ${ }^{60}$ Therefore their intensities are proportional to the values normalized by the biexciton density and are directly comparable with Eq. (12). The experimental results are quantitatively reproduced by the theory, thus the biexciton radiative decay theory on the BP 

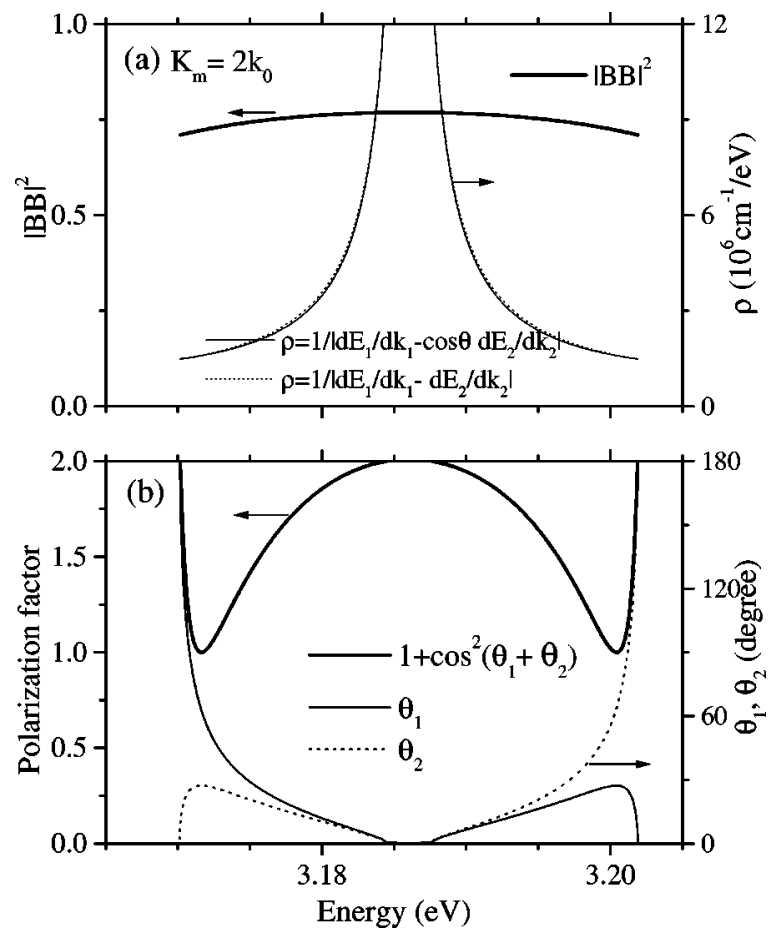

FIG. 18. (a) The transition matrix element $\left|B_{1 s}\left(k_{1}\right) B_{1 s}\left(k_{2}\right)\right|^{2}$ (thick solid curve), the joint density of polariton states $\rho$ $=1 /\left|d E_{1} / d k_{1}-\cos \theta d E_{2} / d k_{2}\right|$ (thin solid curves) as a function of the energy of polaritons emitted from biexcitons of $\mathbf{K}_{m}=2 \mathbf{k}_{0}$ with the internal polariton scattering angle $\theta$ varied from 0 to $\pi$. Here, $\theta=\theta_{1}+\theta_{2}$ and $\theta_{1}=\theta_{o b s}$. The joint density of polariton states calculated with $\theta=0$ (dotted curves) is also shown for comparison. (b) The polarization factor $\left|\mathbf{e}_{1} \cdot \mathbf{e}_{2}\right|^{2}=1+\cos ^{2} \theta$ (thick solid curve), and $\theta_{1}$ (thin solid curve) and $\theta_{2}$ (dotted curve) as a function of the polariton energy.

model is experimentally verified.

Although the transition matrix element between twopolariton and biexciton states is the same for both calculations, the result in Fig. 19(b) shows a much larger intensity at $3.186 \mathrm{eV}$ than in Fig. 19(a). This difference comes from the JDPS. In Fig. 19(b), two polaritons are emitted in the same direction, showing the van Hove singularity at $\theta=0$ with $K_{m}=2 k_{0}$ in Eq. (11). ${ }^{44,61}$ Note that this singularity does not affect the two-photon absorption of $K_{m}=2 k_{0}$ biexcitons. ${ }^{62}$

In the calculations in Fig. 19, errors caused by the approximations of (a) $\theta=\pi$ (antiparallel scattering) and (b) $K_{m}=2 k_{0}$ are smaller than the error bars in the experimental data (uncertainty in estimation of the emission area intensity). In Fig. 19(a), the errors from the antiparallel approximation are within a few percent of that of a precise calculation with $\theta_{d}$. In Fig. 19(b), the experimental results for Fig. 12 (filled circles) are on the condition of $\theta \simeq 0$ with $K_{m}$ varied, while the calculated results are on the condition of $K_{m}$ $=2 k_{0}$ with $\theta$ varied. But both results can be mutually compared since the JDPS with $\theta=0$ (dotted curves) are not much different from that with $\theta$ varied (thin solid curves) in Fig. $18(\mathrm{a})$, and the polarization factor gives a less than $10 \%$ difference between $3.18 \mathrm{eV}$ and $3.192 \mathrm{eV}$ in Fig. 18(b).

To be strict, there are appreciable differences between the experiment and theory both in Figs. 19(a) and 19(b). In Fig.

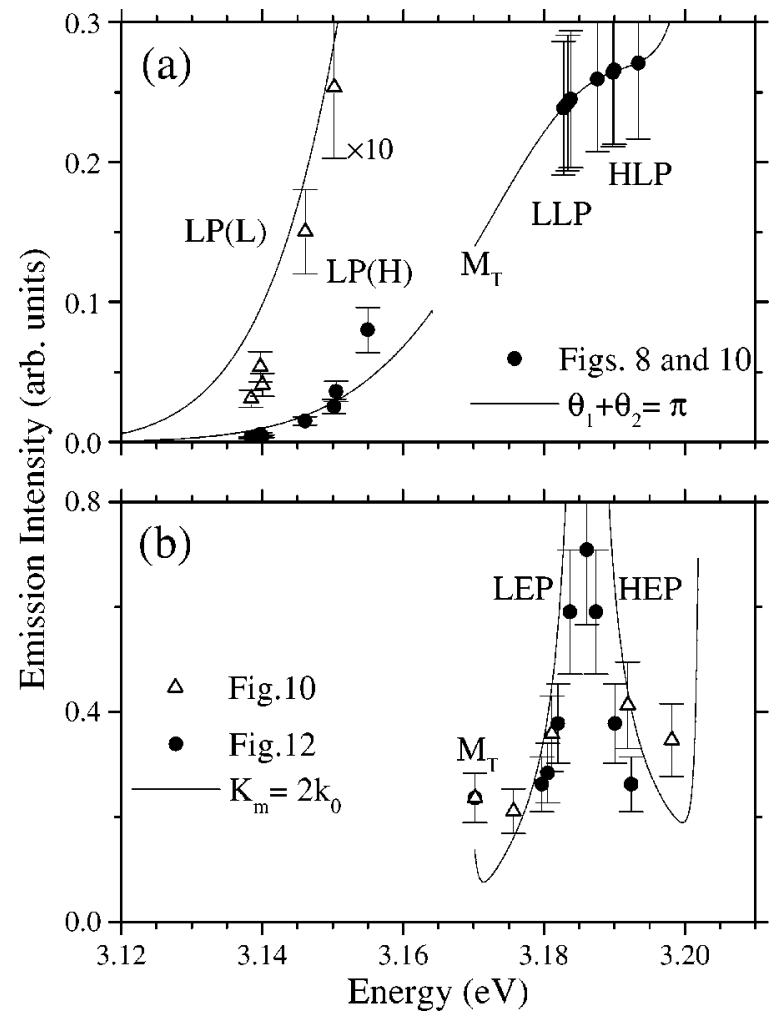

FIG. 19. (a) The biexciton emission intensity as a function of the emitted polariton energy for $K_{m} \sim 0$ biexcitons. Solid curves: calculated results with $\theta_{1}+\theta_{2}=\pi$ (antiparallel emission condition) as the biexciton wave number is varied from $K_{m}=-2 k_{0}$ to $K_{m}$ $=2 k_{0}$ for LBP-LBP and from $K_{m}=-0.89 k_{0}$ to $K_{m}=2 k_{0}$ for UBPLBP. Filled circles $(\mathbf{)})$ : experimental results in Figs. 8 and 10. Open triangles $(\triangle)$ : experimental results magnified ten times. The error bars indicate uncertainty in estimation of the frequencyintegrated intensities. The relative intensity of the $\operatorname{LP}(L)[\mathrm{LP}(H)]$ line with respect to that of the LLP (HLP) line is plotted with the latter intensity normalized to the calculated value. The deviation of the experimental point from the curve at $3.155 \mathrm{eV}$ is partly due to the underestimation of the HLP intensity in Fig. 10(f), where the HLP line is overlapped with the laser scattering (HL). (b) The biexciton emission intensity as a function of the emitted polariton energy for $K_{m} \sim 2 k_{0}$ biexcitons. Solid curves: calculated results at $\mathbf{K}_{m}=2 \mathbf{k}_{0}\left(0.885 \times 10^{6} \mathrm{~cm}^{-1}\right)$ as $\theta_{1}$ is varied from $0^{\circ}$ to $180^{\circ}$. Filled circles $(\mathbf{)})$ : experimental results in Fig. 12. Open triangles $(\triangle)$ : experimental results in Figs. 10(a) and 10(b), where the $M_{T}$ emissions are in the observing direction while the HEP and LEP emissions are observed after internal reflection on the rear surface, so that the HEP and LEP intensities are corrected with the reflectivity. The relative intensity of the LEP(HEP) line with respect to that of the $M_{T}$ line is plotted. The vertical axes in (a) and (b) are scaled in the same unit such that the calculated intensity is mutually compared. For both (a) and (b), the calculated results at $3.17 \mathrm{eV}$ correspond to $M_{T}$ emissions from $\mathbf{K}_{m}=2 \mathbf{k}_{0}$ biexcitons in a backscattering geometry.

19(a), the $\mathrm{LP}(L)$ intensities around $3.14 \mathrm{eV}$ are smaller than the calculated values as shown by the open triangles. This may partly come from the approximation in Eq. (10), where the biexciton wave function is modeled by an envelope function $\Psi(\mathbf{R})$ as explained in Appendix B. In Fig. 19(b), the 
increase of the observed emission intensity toward $3.186 \mathrm{eV}$ is less pronounced than the theoretical curve. This difference comes partly from the finite laser widths, which are not considered in the calculation. Actually the emission intensity is a value integrated over the emission width, which smooths out the divergence at the degenerate energy. As pointed out in Sec. IV B, the elastic scattering of biexcitons should also be responsible for the broadening of the emission lines, and thus for the difference here. ${ }^{63}$

\section{Collisional excitation}

As shown in Figs. 17-19, nearly degenerate emissions are more efficient processes than nondegenerate emissions around the $M_{T}$ energy $(\simeq 3.17 \mathrm{eV})$ when biexcitons are $\mathbf{K}_{m}$-selectively excited. This raises a question as to why nearly degenerate emissions are absent for collisionally excited biexcitons in Fig. 7 in spite of their higher emission efficiency. In order to clarify this quantitatively, the calculation of the emission spectrum is performed for biexcitons distributed in a wide wave-number region as follows. When biexcitons are distributed isotropically at a fixed wave number $K_{m}$ (on the sphere of the radius $K_{m}$ ), the emission spectrum observed from one direction is (Appendix C)

$$
\begin{aligned}
I\left(K_{m}, E_{1}\right) \propto \frac{d \theta_{1}}{d E_{1}} & \\
& \times \frac{k_{1}^{2} \sin \theta_{1}\left(1+\cos ^{2} \theta\right)\left|B_{1 s}\left(k_{1}\right) B_{1 s}\left(k_{2}\right) W_{1,1} C_{1,1}\right|^{2}}{\frac{d E_{1}}{d k_{1}}-\frac{d E_{2}}{d k_{2}} \cos \theta} \\
= & \frac{d k_{z}}{d E_{1}} \frac{\left(1+\cos ^{2} \theta\right)\left|B_{1 s}\left(k_{1}\right) B_{1 s}\left(k_{2}\right) W_{1,1} C_{1,1}\right|^{2}}{\frac{d E_{1}}{d k_{1}} \frac{1}{k_{1}}+\frac{d E_{2}}{d k_{2}} \frac{1}{k_{2}}}
\end{aligned}
$$

where $k_{z}$ is the projection of $\mathbf{k}_{1}$ onto $\mathbf{K}_{m}$, and $W_{1,1}$ and $C_{1,1}$ are generalized forms of $\epsilon^{m}$ and $\int d \mathbf{R} \Psi(\mathbf{R})$ in Eq. (10), which are both defined in Appendix B. Here only the transverse exciton (coupled with the photon) is taken into account as the final state. Then, the total emission spectrum for a biexciton distribution function $f\left(K_{m}\right)$ is obtained by

$$
F(E)=\int d K_{m} I\left(K_{m}, E\right) f\left(K_{m}\right) K_{m}^{2} .
$$

For simplicity, we take $f\left(K_{m}\right)=1$ (constant) and the integration is made from $K_{m}=0$ to $10 \times 10^{6} \mathrm{~cm}^{-1}$. In the above equation, the $k$ dependences of $W_{1,1}$ and $C_{1,1}$ should be included, different from the case for $K_{m} \sim 0$ in Eq. (10). The explicit forms of $W_{1,1}\left(\mathbf{k}_{1}-\mathbf{k}_{2}\right)$ and $C_{1,1}\left(\mathbf{k}_{1}-\mathbf{k}_{2}\right)$ are given in Appendix B. For $C_{1,1}$, however, we assume $C_{1,1}=1$ (constant) since the biexciton wave function is not precisely known. This is equivalent to the assumption that the size of the biexciton wave function is much smaller than $1 / K_{m}$. This approximation will result in an appreciable difference for $K_{m}>5 \times 10^{6} \mathrm{~cm}^{-1}$ but within a factor of $2 .{ }^{33}$ Figure 20 (a) shows the calculated result for $F(E)$ together with the re-
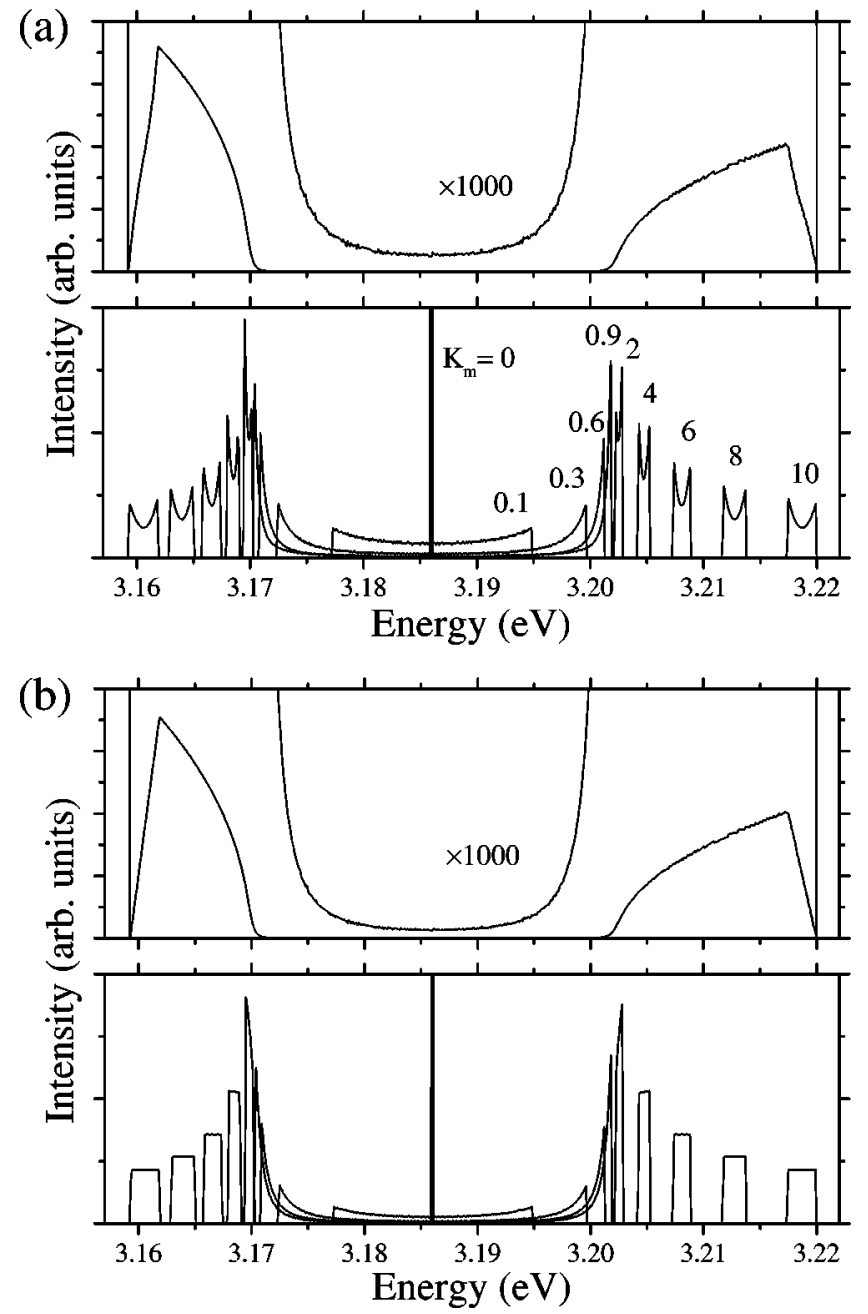

FIG. 20. (a) Top: the calculated emission spectrum $F(E)$ using Eq. (15) of biexcitons distributed homogeneously in the band from $K_{m}=0$ to $10 \times 10^{6} \mathrm{~cm}^{-1}$. The transition into the longitudinal exciton is not considered. Bottom: the calculated emission spectrum $I\left(K_{m}, E\right)$ of biexcitons distributed isotropically at the fixed wave number of $K_{m}=0,0.1,0.3,0.6,0.9,2,4,6,8$, and 10 $\times 10^{6} \mathrm{~cm}^{-1}$. (b) Results of calculation using Eq. (17), i.e., without the matrix element, joint density of polariton states, and polarization factor.

spective contributions $I\left(K_{m}, E\right)$ from $K_{m}=0,0.1,0.3,0.6$, $0.9,2,4,6,8$, and $10 \times 10^{6} \mathrm{~cm}^{-1}$. Since internal reflection and relaxation of the final excitons are not taken into account, the spectrum above $3.186 \mathrm{eV}$ is unrealistic but it helps to see the relation between the $M$-band energy and the exciton energy. The asymmetry of the spectrum $F(E)$ is caused by the increase in the biexciton energy with $K_{m}$, as seen in the blue shift of the center of the $I\left(K_{m}, E\right)$ spectra. As opposed to the larger transition probability demonstrated in Fig. 19 , the emission intensity around the degenerate energy is negligibly small in Fig. 20(a). This is because the effects of the initial biexciton density of states $\left(K_{m}^{2}\right)$ and the final polariton density of states $\left(d k_{z} / d E_{1}\right)$ are superior to the effects of the matrix element $\left(|B B W C|^{2}\right)$ and the JDPS $\left[1 / d E_{1} / d k_{1}-\left(d E_{2} / d k_{2}\right) \cos \theta\right]$, which becomes significant only in the case of $\theta \simeq 0$ and $K_{m} \simeq 2 k_{0}$ (i.e., $\mathbf{k}_{1} \simeq \mathbf{k}_{2}$ ). This is 


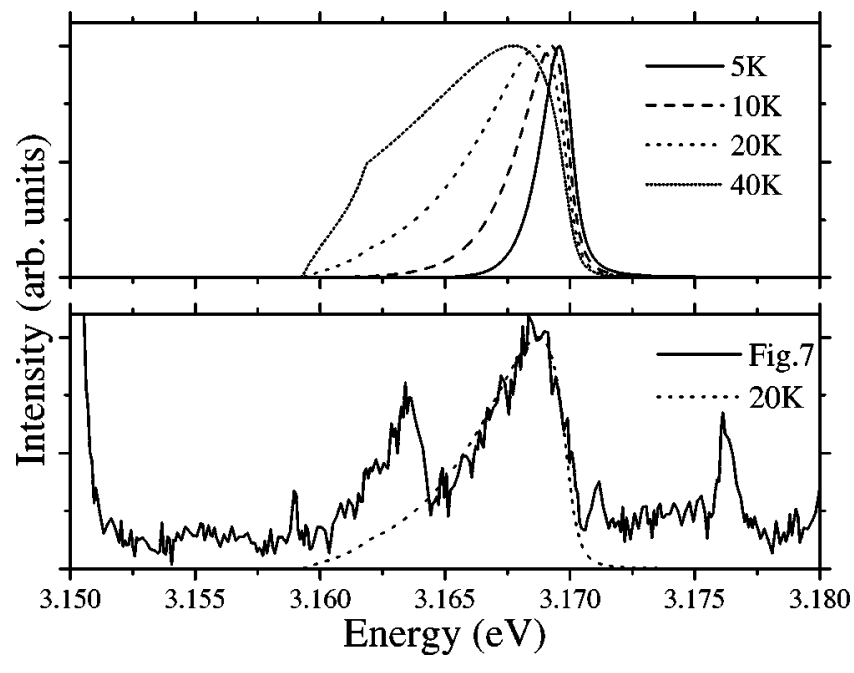

FIG. 21. Top: the calculated emission spectra of biexcitons having Boltzmann distributions of $T=5,10,20$, and $40 \mathrm{~K}$. Bottom: the $M_{T}$ band in Fig. 7 is compared with the calculated spectrum for $T$ $=20 \mathrm{~K}$.

already evident from Figs. 10 and 12: if the emission intensities normalized by the bandwidth $\Delta E$ are compared, the $M_{T}$ intensity is larger than the intensity of nearly degenerate emissions owing to the concentration of final polariton density of states. Note that in Fig. 19 the emission intensities integrated over the total bandwidth are analyzed on the condition of fixed $\Delta K_{m}$ in both theory and experiment.

It is readily shown that the simple calculation without the matrix element (including the polarization factor) and JDPS gives little difference from the rigorous calculation above. Figure 20(b) shows the results with the integrand in Eq. (15) as a constant, i.e., by only taking account of the distribution of the emission energy (final polariton density of states) as follows.

$$
I\left(K_{m}, E_{1}\right) \propto \frac{d k_{z}}{d E_{1}} .
$$

Although $I\left(K_{m}, E\right)$ are different between Figs. 20(a) and 20(b), $F(E)$ are almost identical. The difference is found only around the degenerate energy in the magnified scale by 1000 times.

Figure 21 shows the result for a Boltzmann distribution of $f\left(K_{m}\right)=\exp \left[-\left(K_{m}^{2} / 2 M_{m}\right) / k_{B} T\right]$ with $T=20 \mathrm{~K}$ and $I\left(K_{m}, E\right)$ using Eq. (15), in fair agreement with the experimental results given in Fig. 7. To conclude, the correction by polariton effects ( $B B$ formula) for the $M$-band spectra from thermally distributed biexcitons can be safely neglected for most of the cases. This justifies previous simple analyses of $M$ bands without the matrix element. ${ }^{64}$

\section{B. Two-photon absorption and two-photon emission}

Figure 5 shows that the UP and LP emission intensities decrease as their energy difference increases. In order to explain this behavior quantitatively, both biexciton generation and biexciton radiative decay processes should be treated precisely including polariton effects.

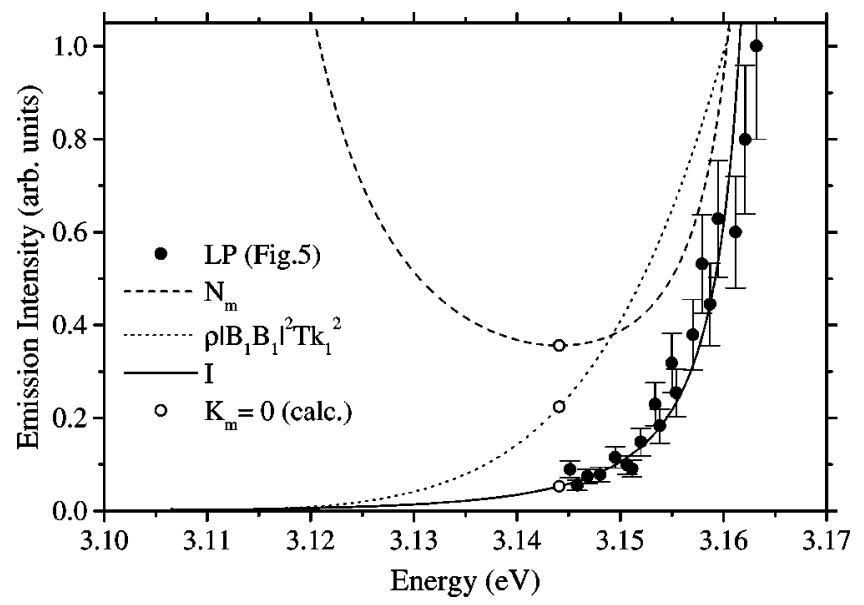

FIG. 22. The LP emission intensity as a function of energy for the experiment (dots) in Fig. 5 and the calculation (solid curves) by Eq. (19). The calculated results are normalized appropriately to fit with the experimental results. The error bars indicate uncertainty in estimation of the frequency-integrated intensities. Also shown are the corresponding biexciton density $N_{m}\left(K_{m}\right)$ (dashed curve) and the emission intensity per biexciton density $\gamma\left(\mathbf{K}_{\mathbf{m}}, E_{1}\right) T\left(E_{1}\right) k_{1}^{2}$ (dotted curve).

The biexciton population density is proportional to the two-photon absorption intensity. For the two-input laser beams of energies $E_{1}^{(0)}$ and $E_{2}^{(0)}$ satisfying $E_{m}=E_{1}^{(0)}$ $+E_{2}^{(0)}$, the biexciton density is given by ${ }^{31}$

$$
\begin{aligned}
N_{m}\left(K_{m}\right) \propto & I_{1} I_{2} T\left(E_{1}^{(0)}\right) T\left(E_{2}^{(0)}\right) \\
& \times \frac{\left|\Omega_{1 s}\right|^{2}\left|\Omega_{1 s}\right|^{2}}{\left|E_{1 s}-E_{1}^{(0)}-i \Gamma_{1 s}\right|^{2}\left|E_{1 s}-E_{2}^{(0)}-i \Gamma_{1 s}\right|^{2}} \\
& \times\left|\epsilon^{m} \int d \mathbf{R} \Psi(\mathbf{R})\right|^{2}
\end{aligned}
$$

where $\Gamma_{1 s}$ is the polarization decay rate of the $Z_{3}-1 s$ exciton, and $I_{1}$ and $I_{2}$ are the intensities of the two excitation beams. Here $\Gamma_{1 s}$ can be neglected because it is less than 0.1 meV. ${ }^{58,59}$ To avoid divergence in the numerical calculation, $\Gamma_{1 s}$ is taken to be $0.03 \mathrm{meV}$. In Eq. (18), the polarization factor is omitted because of the one-dimensional excitation geometry $(\theta=\pi)$.

The observed emission intensity in Fig. 5 is calculated with Eqs. (10) and (18) as

$$
I\left(\mathbf{K}_{m}, E_{1}\right) \propto N_{m}\left(K_{m}\right) \gamma\left(\mathbf{K}_{m}, \mathbf{k}_{1}\right) T\left(E_{1}\right) k_{1}^{2} .
$$

In $\gamma\left(\mathbf{K}_{m}, \mathbf{k}_{1}\right), \rho\left(k_{1}, k_{2}\right) \propto 1 /\left[v_{g}\left(k_{1}\right)+v_{g}\left(k_{2}\right)\right]$ and $\left|\mathbf{e}_{1} \cdot \mathbf{e}_{2}\right|^{2}$ $=2$ (antiparallel scattering), where $v_{g}$ is the polariton group velocity. The experimental result to be compared with this formula is the LP emission intensity in Fig. 5. The UP emission intensity is not suitable because UBP's are subject to relaxation into LBP's during propagation to the surface, ${ }^{47}$ requiring an additional factor in the analysis. Figure 22 shows the LP emission intensity as a function of energy for the experiment and the theory with Eq. (19). The theory reproduces the experimental results fairly well. For a more precise analysis, Eq. (19) should be slightly modified to in- 
clude the effect of how large is the contribution of the observed emission channel in the total radiative decay channels calculated in Sec. VI, and the experimental intensity should be corrected about fluctuations in the excitation laser powers on tuning the energies and in alignment accuracy to overlap the two beams.

An apparent difference between the absorption and emission processes should be noted. The absorption process by Eq. (18) is most efficient when one of the excitation lasers is at the exciton resonance, ${ }^{31}$ while the radiative decay process by Eq. (10) is most efficient at the degenerate two-photon energy, $3.186 \mathrm{eV}$. The different energy dependence between the induced absorption and spontaneous emission processes shown by Eqs. (18) and (10) is due to the difference in the measured quantities. ${ }^{32}$ In both processes, the transition probability between the initial and final states is expressed by the same transition matrix element, $\left|\mathbf{e}_{1} \cdot \mathbf{e}_{2} B_{1 s}\left(k_{1}\right) B_{1 s}\left(k_{2}\right) \epsilon^{m} \int d \mathbf{R} \Psi(\mathbf{R})\right|^{2}$, as in Eq. (10). In the emission process of biexcitons, the time-integrated intensity normalized by the biexciton density is obtained. This is proportional to the quantum efficiency of the biexciton-topolariton transition (the polariton numbers $n_{i}=n_{j}=1$ ), thus the original $|B B|$ formula is retained. In the absorption process, on the other hand, there are two input beams of constant flux densities $I_{1}$ and $I_{2}\left(\mathrm{~W} / \mathrm{cm}^{2}\right)$, and the intensities of the transmitted (diffracted) beams are normalized by $I_{1} I_{2}$ to yield the $\chi^{(3)}$ formula as in Eq. (18). In this case the polariton number density $n_{j}$ is not unity but is given by $n_{j}$ $=I_{j} / \omega_{j} v_{g}\left(k_{j}\right)\left(\mathrm{cm}^{-3}\right)$, where $\omega_{j}$ is the energy $(\mathrm{J})$ of a single polariton and $v_{g}\left(k_{j}\right)$ is the polariton group velocity $(\mathrm{cm} / \mathrm{s})$. Due to the dispersion of $v_{g}\left(k_{j}\right)$, the resonance denominators of the $B$ coefficients are canceled and replaced by those having a sharp exciton resonance as described in Ref. 65.

\section{WAVE-NUMBER DEPENDENCE OF BIEXCITON RADIATIVE LIFETIME}

In order to explain the dynamical behavior of biexcitons observed in Fig. 6, the biexciton radiative decay rate is calculated by integrating the transition probability over all possible radiative decay channels ${ }^{66}$ as follows (Appendix C):

$$
\begin{aligned}
\Gamma\left(K_{m}\right) \propto & \int d \mathbf{k}_{1} d \mathbf{k}_{2} \delta\left(\mathbf{K}_{m}-\mathbf{k}_{1}-\mathbf{k}_{2}\right) \delta\left(E_{m}-E_{1}\right. \\
& \left.-E_{2}\right)\left|\mathbf{e}_{1} \cdot \mathbf{e}_{2}\right|^{2}\left|B_{1 s}\left(k_{1}\right) B_{1 s}\left(k_{2}\right)\right|^{2} \\
& \propto \int d k_{z} \frac{\left|\mathbf{e}_{1} \cdot \mathbf{e}_{2}\right|^{2}\left|B_{1 s}\left(k_{1}\right) B_{1 s}\left(k_{2}\right)\right|^{2}}{\frac{d E_{1}}{d k_{1}} \frac{1}{k_{1}}+\frac{d E_{2}}{d k_{2}} \frac{1}{k_{2}}},
\end{aligned}
$$

where $\mathbf{K}_{m}$ is taken along the $z$ axis and $k_{z}$ is the $z$ component of $\mathbf{k}_{1}$. Decay channels involved are LBP $+\mathrm{LBP}, \mathrm{LBP}+\mathrm{UBP}$, and LBP+LE, where LE represents a longitudinal exciton. Recently found channels into the higher polariton branches are omitted because of their negligibly small intensity. ${ }^{40}$ The polarization factor $\left|\mathbf{e}_{1} \cdot \mathbf{e}_{2}\right|^{2}$ is $1+\cos ^{2} \theta$ for $\mathrm{LBP}+\mathrm{LBP}$ and LBP+UBP and $\sin ^{2} \theta$ for LBP+LE. ${ }^{17}$

Figure 23 shows the calculated $K_{m}$ dependence of the relative radiative decay rate. Here, the generalized Hopfield

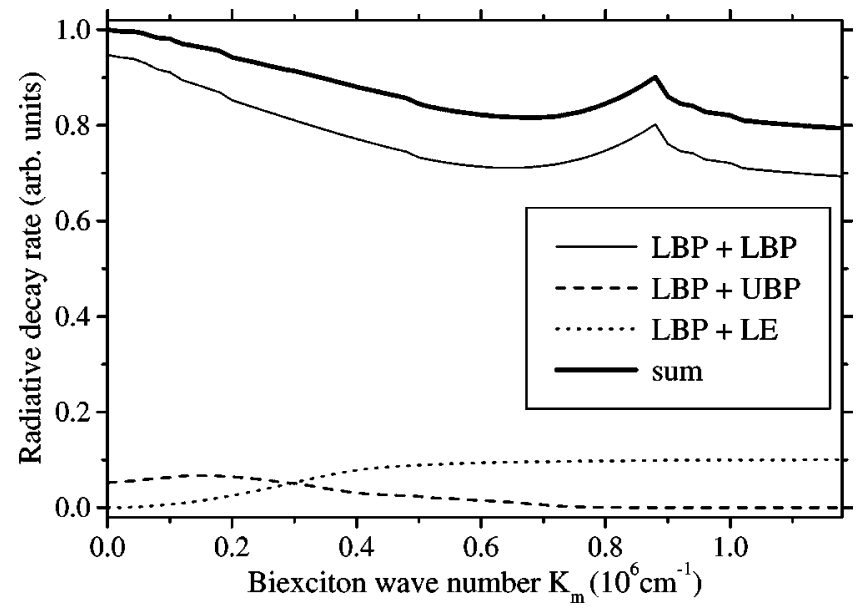

FIG. 23. The $K_{m}$ dependence of the biexciton radiative decay rates due to relaxation into two lower-branch polaritons (LBP $+\mathrm{LBP})$, lower- and upper-branch polaritons (LBP+UBP), and lower-branch polaritons and longitudinal excitons (LBP+LE), calculated with Eq. (15). In Fig. 5, LEP-HEP and $M_{T}$-EX emissions are from the LBP-LBP channel, LP-UP emissions from the LBPUBP channel, and $M_{L}$ emissions from the LBP-LE channel.

coefficients including the $Z_{1,2}$ exciton are used [Eq. (13)] and $B_{1 s}\left(k_{2}\right)$ for LBP+LE is taken to be unity, where $k_{2}$ is the wave number of longitudinal excitons. Compared to the calculation with the one-oscillator model, the LBP+UBP channel is significantly modified, ${ }^{67}$ but the total $K_{m}$ dependence is not altered qualitatively since the contribution of this channel is much smaller than that of the LBP+LBP channel. The decay rate has a local maximum at $K_{m}=2 k_{0}$ because the joint density of polariton states has a singularity in the path of $\mathbf{K}_{m}=\mathbf{k}_{0}+\mathbf{k}_{0}$ as discussed in Sec. V. ${ }^{61}$ The experimental observation of the shorter decaytime (larger decay rate) for $K_{m}=0$ than for $K_{m} \simeq 2 k_{0}$ in Fig. 6 is reproduced by the calculation.

If there are no polariton effects, biexcitons decay into a flat portion of the exciton dispersion for any $K_{m}$, leading to the absence of the $K_{m}$ dependence of the radiative decay rate. In this case the value of the decay rate should be almost equivalent to those for $K_{m}>1.2 \times 10^{6} \mathrm{~cm}^{-1}$ in Fig. 23. In the presence of polariton effects, by contrast, $K_{m}=0$ biexcitons decay into strongly coupled polariton states with a decay rate 1.25 times larger (20\% reduction in the decay time) than in the case of no polariton effects, as evident from the difference between $K_{m}=0$ and $K_{m}>1.2 \times 10^{6} \mathrm{~cm}^{-1}$ in Fig. 23. The larger decay rate is due to the high efficiency of degenerate two-polariton emissions at $K_{m}=0$ and this is the very effect of bipolariton coupling. Here both Hopfield coefficients and JDPS are responsible for the larger decay rate, while the increase of the decay rate at $K_{m}=2 k_{0}$ is mainly due to the singularity of the JDPS. ${ }^{31}$

The observed decay time is consistent with the estimate from the two-photon absorption width at $K_{m}=0 .{ }^{31}$ The $K_{m}$ dependence of a biexciton decay rate is also calculated in Ref. 31 on an exactly solvable BP model. The results in Fig. 23 are slightly different from Fig. 9 of Ref. 31, where the decay rate of $K_{m}=2 k_{0}$ biexcitons is larger than that of $K_{m}$ $=0$ biexcitons. This is because in Ref. 31 the polarization 
factor $\left|\mathbf{e}_{1} \cdot \mathbf{e}_{2}\right|^{2}$ is taken as a constant, not as $1+\cos ^{2} \theta$. The calculation of $\Gamma\left(\mathbf{K}_{m}\right)$ on LBP + LBP with $\left|\mathbf{e}_{1} \cdot \mathbf{e}_{2}\right|^{2}=1$, which corresponds to the polariton polarizations vertical to the plain made from $\mathbf{k}_{1}$ and $\mathbf{k}_{2}$, reproduces almost the same $K_{m}$ dependence as in Fig. 9 of Ref. 31. The factor of $\cos ^{2} \theta$ comes from the polarizations parallel to the plane. At $K_{m}$ $=0,\left|\mathbf{e}_{1} \cdot \mathbf{e}_{2}\right|^{2}=2$ because the angle $\theta$ between $\mathbf{k}_{1}$ and $\mathbf{k}_{2}$ is always $180^{\circ}$. For higher $K_{m}$, the $\cos ^{2} \theta$ factor reduces $\Gamma_{L B P-L B P}\left(\mathbf{K}_{m}\right)$ because $\theta<180^{\circ}$ in average.

The $K_{m}$ dependence of the biexciton absorption width is reported in Ref. 68, where the width at $K_{m}=2 k_{0}$ is larger than at $K_{m}=0$ by a factor of 3 . This is a trend opposite to the present observation and theory, where the radiative lifetime at $K_{m}=2 k_{0}$ is longer than at $K_{m}=0$. In our observation, this experimental fact is confirmed by several samples. The reason for the apparent inconsistency is explained as follows. The absorption width is determined by the phase-relaxation time rather than the radiative lifetime, thus including not only the radiative-relaxation contribution but also intrabandscattering contributions. For $K_{m}=0$, the latter effects can be neglected, but for $K_{m}=2 k_{0}$ or higher wave numbers, a sample-dependent elastic scattering rate affects the absorption width. Similarly, the decay time of $M_{T}$ emissions gives the phase-relaxation time as demonstrated in Ref. 33. For example, it is reported that the decay time of $M_{T}$ emissions ranges from $30-67 \mathrm{ps}$ in different samples, ${ }^{64}$ suggesting sample-dependent phase-relaxation times of biexcitons. Four-wave mixing experiments also show shorter phaserelaxation times for $K_{m}=2 k_{0}$ than for $K_{m}=0{ }^{66}$ Therefore in Ref. 68 it is most likely that the width of the $2 k_{0}$ biexciton gives a larger value than the true radiative width.

Biexcitons are weakly interacting bosons with the possibility of Bose-Einstein condensation (BEC), but there has been no definite evidence so far although several signatures are observed. ${ }^{69}$ The shorter radiative lifetime for $K_{m}=0$ than for higher $K_{m}$ implies that conditions for BEC of biexcitons are severer than previously expected. Note that the BEC will be monitored by the increase of the UP and LP emission intensities for $K_{m}=0$ biexcitons. In particular, since the characteristics of the LP emissions will not be affected by the dense population of excitons and biexcitons because of its photonlike character, the LP emissions will give the clearest signature of the BEC.

\section{CONCLUSIONS}

The radiative transition channels of $\mathbf{K}_{m}$-selectively excited biexcitons in $\mathrm{CuCl}$ are systematically studied from $\mathbf{K}_{m}=0$ to $\mathbf{K}_{m}=2 \mathbf{k}_{0}$, and the $K_{m}$ dependence of all the relaxation channels involving the upper- and lower-branch polaritons is clarified in both one- and three-dimensional scattering geometries. The upper- and lower-branch polariton (UBP and LBP) pair emissions are observed in the range from 3.12 $\mathrm{eV}$ to $3.25 \mathrm{eV}$ and the polariton-dispersion curves are precisely determined in this range. The two-oscillator polariton dispersion including the $Z_{1,2}$ exciton is essential for the correct description of the radiative-decay channels.

Nearly degenerate two-polariton emissions (HLP, LLP, HEP, LEP), which are due to the decay into two lower- branch polaritons (LBP's), are observed for biexcitons both at $\mathbf{K}_{m} \simeq 0$ and $\mathbf{K}_{m} \simeq 2 \mathbf{k}_{0}$ in the three-dimensional scattering condition. Their intensities are larger than those of nondegenerate polariton emissions simultaneously observed, such as upper- and lower-branch polariton (UBP-LBP) pair emissions and lower-branch polariton $\left(M_{T}\right)$ and transverse exciton (EX) pair emissions. Completely degenerate emissions, which are the reverse process of degenerate two-photon excitation of biexcitons, from $2 \mathbf{k}_{0}$ biexcitons show the largest intensity because of the van Hove singularity of the joint density of polariton states.

The relative intensities between LBP-LBP and UBP-LBP emissions and between LBP-LBP and $M_{T}$-EX emissions are rigorously compared on the condition of fixed biexciton wave vectors, showing good agreement with the biexciton radiative-decay theory based on the BP model without any free fitting parameters. Thus the theory is finally verified experimentally for all the polariton branches associated with both ground- and excited-state excitons. The results for the exciton excited states are already reported separately. ${ }^{40}$ The theory depicts that the biexciton radiative-decay rate is determined by the biexciton wave function and the exciton components in final-state polaritons through the excitonexciton interaction, thus it depends explicitly on the Hopfield $B$ coefficients (exciton components). The emission efficiency is largest when the energies of two-polariton emissions are degenerate and have a broad resonance feature as a function of the signal polariton energy. This behavior comes from the Hopfield coefficient, which shows that the exciton component is widely distributed in the polariton branches as large as $\simeq 224 \mathrm{meV}$ (the vacuum Rabi splitting energy for the ground-state exciton in $\mathrm{CuCl}$ ) due to the strong excitonphoton coupling. ${ }^{32}$ This resonance behavior is in remarkable contrast with the two-photon resonant absorption process, the efficiency of which has a sharp resonance enhancement at the exciton energy, ${ }^{31}$ not at the degenerate two-photon energy. This is due to the additional enhancement of the input polariton density determined by the polariton group velocity. ${ }^{32,65}$

The radiative lifetimes of $K_{m} \simeq 0$ biexcitons are found to be shorter than those of high- $K_{m}$ biexcitons. This is due to the high efficiency of (nearly) degenerate two-polariton emissions for $K_{m} \simeq 0$ biexcitons. The result is reproduced by the $K_{m}$ dependence of the biexciton radiative lifetime, calculated by integrating the transition probabilities over all the radiative relaxation channels experimentally identified. The result of calculation shows that polariton effects reduce the lifetime of $K_{m}=0$ biexcitons by $20 \%$ from the case of no polariton effects.

In spite of the high efficiency of the degenerate-emission process, it is proved that for collisionally excited biexcitons the degenerate-emission lines are absent and the $M$ band dominates in the emission spectra. This assures that for most of the cases the emission spectra from thermally distributed biexcitons can be analyzed without the transition matrix element, but only with the initial biexciton and final polariton density of states.

The high efficiency of the degenerate emission process is important because it is quite an effective parametric down- 
conversion process, the efficiency of which is enhanced by both bipolariton coupling and a large size of the biexciton wave function $\left[\int d \mathbf{R} \Psi(\mathbf{R})\right.$ in Eq. (10) $] .{ }^{15}$ This process will offer an attractive system for quantum optics ${ }^{70}$ in view of quantum-mechanical properties of polaritons $s^{71,72}$ and entangled photon pairs due to the exciton-exciton Coulomb correlation. ${ }^{73}$

The present experimental results show a clear evidence that near $K_{m}=0$ biexcitons behave as bipolaritons, i.e., underlying polariton states determine the lifetime of near $K_{m}$ $=0$ biexcitons. In this context, the term "bipolariton" does not refer to the internal structure of the biexciton wave function. So far it has been difficult to tell polariton modification in the biexciton internal structure itself ${ }^{74}$ which is a delicate problem even for the present BP model because the biexciton wave function is not precisely known. Further investigation of $K_{m} \sim 0$ biexcitons and rigorous comparison with theories are necessary to reveal the influence of the light on the very internal structure of the biexciton wave function.

\section{ACKNOWLEDGMENTS}

We wish to thank A. L. Ivanov for helpful discussions and M. Kuwata-Gonokami for showing unpublished work on $K_{m} \sim 0$ biexcitons. We also appreciate valuable discussions with N. Nagasawa and S. V. Nair.

\section{APPENDIX A}

From the simple algebra of Eqs. (1) and (2),

$$
E_{L, l}^{2}=\frac{1}{2}\left[E_{L}^{\prime 2}+E_{l}^{\prime 2} \pm \sqrt{\left(E_{L}^{\prime 2}-E_{l}^{\prime 2}\right)^{2}+4 \frac{\alpha_{1 s} \alpha_{Z}}{\varepsilon_{b}^{2}} E_{T}^{2} E_{t}^{2}}\right] .
$$

Thus, the longitudinal energies $E_{L, l}$ for the two-oscillator polariton dispersion are shifted from the one-oscillator cases $E_{L, l}^{\prime}$.

\section{APPENDIX B}

The full expressions of the biexciton radiative-decay theory are given in Ref. 39 and the angle dependence is given in Ref. 17 as summarized below. The transition rate for biexcitons $\mathbf{K}_{m} \rightarrow$ polariton pairs $\mathbf{k}_{1}+\mathbf{k}_{2}\left(E_{m}=E_{1}+E_{2}\right)$ is given by

$$
\begin{aligned}
\gamma\left(\mathbf{K}_{m}, E_{1}\right)= & \frac{2 \pi}{\hbar} \rho\left(\mathbf{k}_{1}, \mathbf{k}_{2}\right)\left|\mathbf{e}_{1} \cdot \mathbf{e}_{2}\right|^{2} \\
& \times\left|\sum_{i, j} B_{i}\left(k_{1}\right) B_{j}\left(k_{2}\right) W_{i, j} C_{i, j}\right|^{2},
\end{aligned}
$$

where $\rho$ is the JDPS given by

$$
\rho\left(\mathbf{k}_{1}, \mathbf{k}_{2}\right)=\frac{1}{\frac{d E_{1}}{d k_{1}}-\frac{d E_{2}}{d k_{2}} \cos \theta}
$$

with the internal scattering angle $\theta$ between $\mathbf{k}_{1}$ and $\mathbf{k}_{2}$, $\left|\mathbf{e}_{1} \cdot \mathbf{e}_{2}\right|^{2}$ is the polarization factor given by $1+\cos ^{2} \theta$ for transverse excitons, ${ }^{17} B_{n}$ is the generalized Hopfield coeffi- cient ( $n s$ exciton component), ${ }^{32}$ and $W_{i, j}\left(=E_{m}-E_{i s}-E_{j s}\right)$ is the Coulomb interaction between the $i s$ and $j s$ excitons. The projection of the biexciton wave function onto exciton excited states reads

$$
\begin{aligned}
C_{i, j}\left(\frac{\mathbf{k}_{1}-\mathbf{k}_{2}}{2}\right)= & \int d \mathbf{R} d \mathbf{r}_{1} d \mathbf{r}_{2} \Gamma^{*}\left(\mathbf{R}, \mathbf{r}_{1}, \mathbf{r}_{2}\right) \phi_{i}\left(\mathbf{r}_{1}\right) \phi_{j}\left(\mathbf{r}_{2}\right) \\
& \times e^{i \frac{\mathbf{k}_{1}-\mathbf{k}_{2}}{2}} \mathbf{R},
\end{aligned}
$$

where $\phi_{n}$ is the $n s$ exciton wave function. The biexciton wave function is expressed by

$$
\Gamma\left(\mathbf{R}, \mathbf{r}_{1}, \mathbf{r}_{2}\right)=\sum_{i, j} \sum_{\mathbf{k}} C_{i, j}(\mathbf{k}) \phi_{i}\left(\mathbf{r}_{1}\right) \phi_{j}\left(\mathbf{r}_{2}\right) e^{i \mathbf{k R}} .
$$

For biexciton emissions into the ground-state exciton polaritons [Eq. (15)], the following approximate expressions hold:

$$
\begin{aligned}
& \gamma\left(\mathbf{K}_{m}, E_{1}\right) \simeq \frac{2 \pi}{\hbar} \rho\left(\mathbf{k}_{1}, \mathbf{k}_{2}\right)\left|\mathbf{e}_{1} \cdot \mathbf{e}_{2}\right|^{2} \\
& \times\left|B_{1 s}\left(k_{1}\right) B_{1 s}\left(k_{2}\right) W_{1,1} C_{1,1}\right|^{2}, \\
& \Gamma\left(\mathbf{R}, \mathbf{r}_{1}, \mathbf{r}_{2}\right) \simeq \sum_{\mathbf{k}} C_{1,1}(\mathbf{k}) \phi_{1}\left(\mathbf{r}_{1}\right) \phi_{1}\left(\mathbf{r}_{2}\right) e^{i \mathbf{k R}} \\
&= \Psi(\mathbf{R}) \phi_{1}\left(\mathbf{r}_{1}\right) \phi_{1}\left(\mathbf{r}_{2}\right), \\
& C_{1,1}\left(\frac{\mathbf{k}_{1}-\mathbf{k}_{2}}{2}\right) \simeq \int d \mathbf{R} \Psi *(\mathbf{R}) e^{i} \frac{\mathbf{k}_{1}-\mathbf{k}_{2}}{2} \mathbf{R}, \\
& W_{1,1}=E_{m}\left(\mathbf{K}_{m}\right)-E_{1 s}\left(\mathbf{k}_{1}\right)-E_{1 s}\left(\mathbf{k}_{2}\right) \\
&=E_{m}\left(K_{m}=0\right)+\frac{\hbar^{2}\left|\mathbf{k}_{1}+\mathbf{k}_{2}\right|^{2}}{4 M_{1 s}}-E_{1 s}\left(k_{1}=0\right) \\
&-\frac{\hbar^{2} k_{1}^{2}}{2 M_{1 s}}-E_{1 s}\left(k_{2}=0\right)-\frac{\hbar^{2} k_{2}^{2}}{2 M_{1 s}} \\
&=-\epsilon^{m}-\frac{\hbar^{2}\left|\mathbf{k}_{1}-\mathbf{k}_{2}\right|^{2}}{4 M_{1 s}},
\end{aligned}
$$

where $M_{1 s}$ is the $1 s$-exciton mass. This form for $W_{1,1}$ is given in Ref. 31.

Further, for the emissions from $K_{m} \sim 0$ biexcitons such as nearly degenerate polariton emissions [Eq. (10)], all the $k$ dependences can be neglected such that

$$
C_{1,1} \simeq \int d \mathbf{R} \Psi *(\mathbf{R})
$$

and

$$
W_{1,1} \simeq-\epsilon^{m} .
$$

In fact, the $k$ dependence of $W_{1,1}$ gives a correction of $1 \%$ in the transition rate for $\left|\mathbf{K}_{m}\right|<2 k_{0}$.

In the approximation of parallel $(\theta=0)$ and antiparallel $(\theta=\pi)$ emissions (one-dimensional scattering condition) [Eq. (19)], the polarization factor $\left|\mathbf{e}_{1} \cdot \mathbf{e}_{2}\right|^{2}$ can also be set as a constant. 


\section{APPENDIX C}

The integration of Eqs. (14), (15), and (21) is performed as follows. In the cylindrical coordinate with $\mathbf{K}_{m} \| \mathbf{k}_{z}$ and $F\left(\mathbf{k}_{1}, \mathbf{k}_{2}\right)=\left|\mathbf{e}_{1} \cdot \mathbf{e}_{2}\right|^{2}\left|B_{1 s}\left(k_{1}\right) B_{1 s}\left(k_{2}\right)\right|^{2}$,

$$
\begin{aligned}
\Gamma\left(K_{m}\right) \propto & \int d \mathbf{k}_{1} d \mathbf{k}_{2} \delta\left(\mathbf{K}_{m}-\mathbf{k}_{1}-\mathbf{k}_{2}\right) \\
& \times \delta\left(E_{m}-E_{1}-E_{2}\right) F\left(\mathbf{k}_{1}, \mathbf{k}_{2}\right) \\
= & \int k_{r} d k_{r} d k_{z} d \phi \delta\left(E_{m}-E\right) F\left(\mathbf{k}_{1}, \mathbf{K}_{m}-\mathbf{k}_{1}\right) \\
= & 2 \pi \int k_{r} d E d k_{z} \frac{\delta\left(E_{m}-E\right)}{\frac{\partial E}{\partial k_{r}}} F \\
= & 2 \pi \int k_{r} d k_{z} \frac{F}{\frac{d E_{1}}{d k_{1}} \frac{\partial k_{1}}{\partial k_{r}}+\frac{d E_{2}}{d k_{2}} \frac{\partial k_{2}}{\partial k_{r}}} \\
= & 2 \pi \int d k_{z} \frac{F}{\frac{d E_{1}}{d k_{1}} \frac{1}{k_{1}}+\frac{d E_{2}}{d k_{2}} \frac{1}{k_{2}}},
\end{aligned}
$$

yielding Eq. (21). In the spherical coordinate with $\mathbf{K}_{m} \| \mathbf{k}_{z}$ and the polar angle $\theta_{1,2}$ between $\mathbf{K}_{m}$ and $\mathbf{k}_{1,2}$,

$$
\begin{aligned}
\Gamma\left(K_{m}\right) & \propto \int k_{1}^{2} \sin \theta_{1} d \theta_{1} d k_{1} d \phi \delta\left(E_{m}-E\right) F \\
= & 2 \pi \int d \theta_{1} \frac{k_{1}^{2} \sin \theta_{1} F}{\frac{d E_{1}}{d k_{1}}+\frac{d E_{2}}{d k_{2}} \frac{\partial k_{2}}{\partial k_{1}}} \\
= & 2 \pi \int d \theta_{1} \frac{k_{1}^{2} \sin \theta_{1} F}{\frac{d E_{1}}{d k_{1}}-\frac{d E_{2}}{d k_{2}} \cos \theta}
\end{aligned}
$$

where $\theta=\theta_{1}+\theta_{2}$. This gives the same result as Eq. (C1). In the case of antiparallel emissions $(\theta=\pi)$,

$$
\Gamma\left(K_{m}\right) \propto \Delta \Omega \frac{k_{1}^{2}}{v_{g}\left(k_{1}\right)+v_{g}\left(k_{2}\right)} F,
$$

*Present address: Department of Physics, Faculty of Science, Univ. of Tokyo, 7-3-1 Hongo, Bunkyo, Tokyo 113-0033, Japan.

†Address for research project: Department of Applied Chemistry, Keio University, 3-14-1 Hiyoshi, Kohoku-ku, Yokohama 2238522, Japan.

Permanent address: Institute of Physics, University of Tsukuba, Tsukuba 305-8571, Japan.

${ }^{\S}$ Present address: 1-19-12 Fujimoto, Kokubunji, Tokyo 185-0031, Japan.

${ }^{1}$ M. Ueta, H. Kanzaki, K. Kobayashi, Y. Toyozawa, and E. Hanamura, Excitonic Processes in Solids, Springer Series in SolidState Sciences Vol. 60 (Springer, Berlin, 1986), Chaps. 2 and 3.

${ }^{2}$ J. J. Hopfield, Phys. Rev. 112, 1555 (1958); 182, 945 (1969).

${ }^{3}$ O. Akimoto and E. Hanamura, J. Phys. Soc. Jpn. 33, 1537 (1972).

${ }^{4}$ W.F. Brinkman, T.M. Rice, and B. Bell, Phys. Rev. B 8, 1570 (1973).

${ }^{5}$ M.A. Lee, P. Vashishta, and R.K. Kalia, Phys. Rev. Lett. 51, 2422 (1983).

${ }^{6}$ I.B. Talanina, M.A. Collins, and V.M. Agranovich, Phys. Rev. B 49, 1517 (1994).

${ }^{7}$ C. Weisbuch, M. Nishioka, A. Ishizawa, and Y. Arakawa, Phys. Rev. Lett. 69, 3314 (1992). For a review, see G. Khitrova, H.M. Gibbs, F. Jahnke, M. Kira, and S.W. Koch, Rev. Mod. Phys. 71, 1591 (1999).

${ }^{8}$ F. Quochi, G. Bongiovanni, A. Mura, J.L. Staehli, B. Deveaud, R.P. Stanley, U. Oesterle, and R. Houdré, Phys. Rev. Lett. 80, 4733 (1998).

${ }^{9}$ M. Kuwata-Gonokami, S. Inouye, H. Suzuura, M. Shirane, R. Shimano, T. Someya, and H. Sakaki, Phys. Rev. Lett. 79, 1341 (1997).

${ }^{10}$ Th. Östreich, K. Schönhammer, and L.J. Sham, Phys. Rev. B 58, 12920 (1998).
${ }^{11}$ S. Savasta and R. Girlanda, Phys. Rev. B 59, 15409 (1999).

${ }^{12}$ F. Tassone and Y. Yamamoto, Phys. Rev. B 59, 10830 (1999).

${ }^{13}$ U. Neukirch, S.R. Bolton, N.A. Fromer, L.J. Sham, and D.S. Chemla, Phys. Rev. Lett. 84, 2215 (2000).

${ }^{14}$ M. Sava, F. Quochi, C. Ciuti, U. Oesterle, J.L. Staehli, B. Deveaud, G. Bongiovanni, and A. Mura, Phys. Status Solidi B 221, 157 (2000).

${ }^{15}$ E. Hanamura, Solid State Commun. 12, 951 (1973); A.A. Gogolin and E.I. Rashba, Pis'ma Zh. Éksp. Teor. Fiz. 17, 690 (1973) [JETP Lett. 17, 478 (1973)]; K. Cho, Opt. Commun. 8, 412 (1973).

${ }^{16}$ G.M. Gale and A. Mysyrowicz, Phys. Rev. Lett. 32, 727 (1974).

${ }^{17}$ F. Henneberger and J. Voigt, Phys. Status Solidi B 76, 313 (1976); F. Henneberger, K. Henneberger, and J. Voigt, ibid. 83, 439 (1977).

${ }^{18}$ V. May and J. Röseler, Phys. Status Solidi B 102, 533 (1980).

${ }^{19}$ A.L. Ivanov, H. Haug, and L.V. Keldysh, Phys. Rep. 296, 237 (1998); A.L. Ivanov, in Microscopic Theory of Semiconductors: Quantum Kinetics, Confinement and Lasers, edited by S.W. Koch (World Scientific, Singapore, 1996), pp. 359-399.

${ }^{20}$ B. Hönerlage, R. Lévy, J.B. Grun, C. Klingshirn, and K. Bohnert, Phys. Rep. 124, 161 (1985).

${ }^{21}$ T. Mita, K. Sotome, and M. Ueta, J. Phys. Soc. Jpn. 48, 496 (1980).

${ }^{22}$ Y. Nozue, T. Itoh, and M. Ueta, J. Phys. Soc. Jpn. 44, 1305 (1978); Y. Nozue, N. Miyahara, S. Takagi, and M. Ueta, Solid State Commun. 38, 1199 (1981).

${ }^{23}$ B. Hönerlage, U. Rössler, D.P. Vu, A. Bivas, and J.B. Grun, Phys. Rev. B 22, 797 (1980); Y. Nozue, J. Phys. Soc. Jpn. 51, 1840 (1982).

${ }^{24}$ M. Ojima, Y. Oka, T. Kushida, and S. Shionoya, Solid State Commun. 24, 845 (1977). 
${ }^{25}$ A. Maruani, J.L. Oudar, E. Batifol, and D.S. Chemla, Phys. Rev. Lett. 41, 1372 (1978); A. Maruani and D.S. Chemla, Phys. Rev. B 23, 841 (1981).

${ }^{26}$ Y. Masumoto and S. Shionoya, J. Phys. Soc. Jpn. 49, 2236 (1980); Solid State Commun. 38, 865 (1981).

${ }^{27}$ N. Nagasawa, M. Kuwata, E. Hanamura, T. Itoh, and A. Mysyrowicz, Appl. Phys. Lett. 55, 1999 (1989).

${ }^{28}$ N. Peyghambarian, H.M. Gibbs, M.C. Rushford, and D. Weinberger, Phys. Rev. Lett. 51, 1692 (1983).

${ }^{29}$ R. Shimano and M. Kuwata-Gonokami, Phys. Rev. Lett. 72, 530 (1994).

${ }^{30}$ K. Ema and M. Kuwata-Gonokami, Phys. Rev. Lett. 75, 224 (1995).

${ }^{31}$ A.L. Ivanov, M. Hasuo, N. Nagasawa, and H. Haug, Phys. Rev. B 52, 11017 (1995). They obtained the biexciton radiative width of $24-36 \mu \mathrm{eV}$, corresponding to about $20-30 \mathrm{ps}$ in decay time.

${ }^{32}$ E. Tokunaga, A.L. Ivanov, S.V. Nair, and Y. Masumoto, Phys. Rev. B 63, 233203 (2001).

${ }^{33}$ K. Kurihara, E. Tokunaga, M. Baba, and M. Matsuoka, Phys. Rev. B 52, 8179 (1995).

${ }^{34}$ E. Tokunaga, M. Baba, K. Kurihara, and M. Matsuoka, Prog. Cryst. Growth Charact. Mater. 33, 149 (1996).

${ }^{35}$ M. Kuwata-Gonokami, R. Shimano, J. Iwamatsu, H. Akiyama, T. Kuga, and M. Matsuoka, Phys. Status Solidi B 159, 347 (1990).

${ }^{36}$ M. Cardona, Phys. Rev. 129, 69 (1963).

${ }^{37}$ S. Suga, K. Cho, and M. Bettini, Phys. Rev. B 13, 943 (1976); S. Suga, K. Cho, Y. Niji, J.C. Merle, and T. Sauder, ibid. 22, 4931 (1980).

${ }^{38}$ T. Itoh and T. Suzuki, J. Phys. Soc. Jpn. 45, 1939 (1978).

${ }^{39}$ E. Tokunaga, A.L. Ivanov, S.V. Nair, and Y. Masumoto, Phys. Rev. B 59, 7837 (1999).

${ }^{40}$ E. Tokunaga, A.L. Ivanov, S.V. Nair, and Y. Masumoto, J. Lumin. 87-89, 216 (2000); Phys. Status Solidi B 221, 359 (2000).

${ }^{41}$ M. Inoue and E. Hanamura, J. Phys. Soc. Jpn. 41, 1273 (1976).

${ }^{42}$ H. Akiyama, M. Kuwata, T. Kuga, and M. Matsuoka, Phys. Rev. B 39, 12973 (1989); H. Akiyama, T. Kuga, M. Matsuoka, and M. Kuwata-Gonokami, ibid. 42, 5621 (1990).

${ }^{43}$ D.P. Vu, A. Bivas, B. Hönerlage, and J.B. Grun, Phys. Status Solidi B 86, 159 (1978).

${ }^{44}$ A.L. Ivanov and H. Haug, Phys. Status Solidi B 188, 61 (1995).

${ }^{45}$ The sum of the UP and LP energies at $K_{m}=0$ is $6.373 \mathrm{eV}$, which is slightly larger than the biexciton energy of $6.372 \mathrm{eV}$. This is probably because the two laser energies are not completely degenerate such that $K_{m}$ is not exactly zero. In this case, the lines split and the $\operatorname{UP}(H)$ and $\operatorname{LP}(H)$ lines are predominantly observed. More precise experimental energies for the UP and LP lines at $K_{m}=0$ are estimated to be rather close to $3.2275 \mathrm{eV}$ and $3.1445 \mathrm{eV}$. The parameter values of $E_{t}=3.267 \mathrm{eV}$ and $E_{l}^{\prime}$ $=3.289 \mathrm{eV}$ reproduce these energies at $K_{m}=0$, giving as good a fit as the values in Table I.

${ }^{46}$ Preliminary results on $K_{m}=0$ biexciton emissions are reported in K. Kurihara, Ph.D. thesis, University of Tokyo, 1995; K. Kurihara, E. Tokunaga, M. Baba, and M. Matsuoka, in Excitonic Processes in Condensed Matter, edited by Jai Singh, SPIE Proceedings No. 2362, 1995), pp. 449-458.

${ }^{47}$ F. Vallee, F. Bogani, and C. Flytzanis, Phys. Rev. Lett. 66, 1509 (1991).

${ }^{48}$ E. Tokunaga, K. Kurihara, M. Baba, and M. Matsuoka (unpublished).
${ }^{49}$ M. Ueta, T. Goto, and T. Yashiro, J. Phys. Soc. Jpn. 20, 1022 (1965).

${ }^{50}$ T. Anzai, T. Goto, and M. Ueta, J. Phys. Soc. Jpn. 38, 774 (1975).

${ }^{51}$ Y. Segawa, Y. Aoyagi, and S. Namba, Solid State Commun. 32, 229 (1979).

${ }^{52}$ E. Ostertag, Phys. Rev. Lett. 45, 372 (1980).

${ }^{53}$ W.C. Tait, Phys. Rev. B 5, 648 (1972).

${ }^{54}$ F. Meseguer, J.C. Merle, and M. Cardona, Solid State Commun. 50, 709 (1984).

${ }^{55}$ The calculated value for $2 k_{0}$ from the parameters in Table I is $0.885 \times 10^{6} \mathrm{~cm}^{-1}$.

${ }^{56}$ D. Fröhlich, P. Köhler, W. Nieswand, and E. Mohler, Phys. Status Solidi B 167, 147 (1991).

${ }^{57}$ D.L. Mills and E. Burstein, Phys. Rev. 188, 1465 (1969).

${ }^{58}$ Y. Masumoto, S. Shionoya, and T. Takagahara, Phys. Rev. Lett. 51, 923 (1983).

${ }^{59}$ T. Ikehara and T. Itoh, Phys. Rev. B 44, 9283 (1991); T. Ikehara, Ph.D. thesis, Tohoku University, 1993.

${ }^{60}$ The HEP and LEP intensities in Fig. 10 are corrected about internal reflection.

${ }^{61}$ The singularity for $\mathbf{K}_{m}=\mathbf{k}_{0}+\mathbf{k}_{0}$ is shown in Fig. 14, where $d E_{1} / d K_{m}\left[=1 /\left(d k_{1} / d E_{1}-d k_{2} / d E_{2}\right)\right] \rightarrow \infty$ for $K_{m} \rightarrow 2 k_{0}$, and $\rho \propto d k_{1} /\left.d E\right|_{E=E_{m}}=1 /\left(d E_{1} / d k_{1}-d E_{2} / d k_{2}\right) \rightarrow \infty\left(k_{1,2} \rightarrow k_{0}\right)$.

${ }^{62}$ In contrast to the emission process, the two-photon absorption intensity is not different between the collinear excitation $\mathbf{k}_{0}$ $+\mathbf{k}_{0}$ and the antiparallel excitation $\mathbf{k}_{0}-\mathbf{k}_{0}$ (Ref. 31), because the number of transition channels for a fixed biexciton width $\Delta K_{m}$ is different between $\mathbf{K}_{m}=\mathbf{k}_{0}-\mathbf{k}_{0}$ and $\mathbf{K}_{m}=\mathbf{k}_{0}+\mathbf{k}_{0}$ while it is the same for a fixed-input laser width $\Delta E$.

${ }^{63}$ The finite biexciton radiative width $\gamma_{m}$, which is not taken into account in the present expression of the JDPS, also relaxes the divergent behavior as given in Ref. 31. Roughly, it is limited by $\rho=\int d k_{1}(1 / 2) \gamma_{m} /\left(E_{m}-E_{1}-E_{2}\right)^{2}+(1 / 4) \gamma_{m}^{2} \sim 2 \Delta k_{1} / \gamma_{m}$ for $\mathbf{k}_{1}=\mathbf{k}_{2}=\mathbf{k}_{0}$.

${ }^{64}$ T. Ikehara and T. Itoh, Solid State Commun. 79, 755 (1991).

${ }^{65}$ See Eqs. (41)-(43) of Ref. 31.

${ }^{66}$ A similar calculation was done on the modified GOS model (Ref. 17) by M. Kuwata-Gonokami, H. Suzuura, T. Ogasawara, R. Shimano, K. Ema, in Proceedings of the 47th Annual Meeting of Japanese Physical Society (Tokyo, 1992), Vol. 47, No. 2, p. 323; T. Ogasawara and H. Suzuura, B.S. thesis, University of Tokyo, 1992.

${ }^{67}$ The contribution of LBP+UBP at $K_{m}=0$ is significantly increased in Fig. 23 by the inclusion of the $Z_{1,2}$ exciton, while the one-oscillator model yields a nearly zero transition rate into LBP + UBP at $K_{m}=0$ due to the larger detuning of LP and UP energies from the $Z_{3}-1 s$ exciton resonance.

${ }^{68}$ M. Hasuo, N. Nagasawa, and T. Itoh, Opt. Commun. 85, 219 (1991).

${ }^{69}$ M. Hasuo, N. Nagasawa, T. Itoh, and A. Mysyrowicz, Phys. Rev. Lett. 70, 1303 (1993), and references therein.

${ }^{70}$ As shown in Fig. 12, the degenerate emissions are significantly broadened compared with the input lasers due to sparse distributions of final polariton states. This allows a precise selection of pair-polariton modes originating from the same biexcitons by spectrally filtering the emission bands. Negligibly small polariton scattering rates are also advantageous. 
${ }^{71}$ M. Artoni and J.L. Birman, Phys. Rev. B 44, 3736 (1991); Opt. Commun. 89, 324 (1992).

${ }^{72}$ A. Quattropani, L.C. Andreani, and F. Bassani, Nuovo Cimento D 7, 55 (1986); P. Schwendimann, A. Quattropani, and Z. Hradil, ibid. 15, 1421 (1993).

${ }^{73}$ S. Savasta, G. Martino, and R. Girlanda, Solid State Commun. 111, 495 (1999).

${ }^{74}$ A.L. Ivanov and H. Haug, Phys. Rev. Lett. 74, 438 (1995). 